\title{
Sound Velocities at High Pressure and Temperature and Their Geophysical Implications
}

\author{
Thomas S. Duffy and Thomas J. Ahrens \\ Seismological Laboratory, California Institute of Technology, Pasadena
}

\begin{abstract}
Temperature coefficients of compressional and bulk sound velocities at pressures on the order of $100 \mathrm{GPa}$ are obtained from Hugoniot sound velocity measurements for solid $\mathrm{Al}, \mathrm{W}, \mathrm{Cu}, \mathrm{Ta}$ and $\mathrm{Mg}_{2} \mathrm{SiO}_{4}$. The Hugoniot velocities are compared to third-order finite strain extrapolations of velocities along the principal isentrope using ultrasonically determined coefficients. At low pressure, where thermal effects are minor, good agreement is found between the Hugoniot velocities and finite strain extrapolations. At high pressures, differences in velocities and temperatures are used to constrain temperature coefficients of velocity. For all materials studied except $W$, the temperature coefficients of velocity at pressures above 1 Mbar are a factor of 2 to 8 smaller in magnitude than zero-pressure values. In shock-melted materials, the Hugoniot sound velocities are close to finite strain velocities calculated from low-pressure properties of the solid phase for $\mathrm{Mo}, \mathrm{Ta}, \mathrm{Pb}, \mathrm{Fe}$, and alkali halides. The temperature coefficient determined for the high-pressure phases of forsterite above $100 \mathrm{GPa}\left(\left|\left(\partial V_{P} / \partial T\right)_{P}\right|=0.1 \pm 0.1 \mathrm{~m} / \mathrm{s} / \mathrm{K}\right)$ is in agreement with estimates based on elastic and thermodynamic properties for the Earth. Our results indicate that $\left|\left(\partial V_{P} / \partial T\right)_{P}\right|$ is a decreasing function of pressure in contrast to residual sphere studies which suggest $\left|\left(\partial V_{P} / \partial T\right)_{P}\right|$ is nearly constant with depth in the Earth. In combination with mineral physics estimates of thermal expansivity at high pressure, it is estimated that $\left(\partial V_{P} / \partial \rho\right)_{P}=2$ $(\mathrm{km} / \mathrm{s}) /\left(\mathrm{g} / \mathrm{cm}^{3}\right)$ for $P>100 \mathrm{GPa}$, with acceptable values ranging from 0 to 8 . This overlaps the range of estimated lower mantle values based on seismic and geodetic data. Tomographic and free oscillation data require large increases in the parameter $\nu=\left(\partial \ln V_{S} / \partial \ln V_{P}\right)_{P}$ under lower mantle conditions, relative to laboratory values. Availáble data for tungsten and aluminum yield $\nu$ values along the Hugoniot that are consistent with zero-pressure values for these materials, although uncertainties are $\pm 50 \%$. Temperature coefficients of velocity at high pressure are used to make improved estimates of the magnitude of thermal heterogeneities sampled by seismic tomography. Long-wavelength compressional velocity anomalies at pressures in the 100-127 GPa range (2271$2891 \mathrm{~km}$ depth) in the lower mantle correspond to temperature variations of $120 \pm 100 \mathrm{~K}$, whereas those in the $D^{\prime \prime}$ region are likely to be a factor of 3 to 4 larger.
\end{abstract}

\section{INTRODUCTION}

The effect of both pressure and temperature on elastic wave velocities must be understood in order to properly interpret the seismologically observed structure of the Earth. For example, recent advances in seismic imaging have produced detailed three-dimensional maps of seismic velocity variations in the mantle and core. Velocity anomalies associated with subducting slabs have been studied using the residual-sphere technique. The observed anomalies may be due to temperature, phase, composition, or possibly noise in the data set. In order to assess the results, the relationship between sound velocities and these various factors must be understood. Similar factors must be considered when sound velocities are compared to the seismically determined radial structure of the Earth in order to constrain compositional models. Laboratory elasticity data have been generally used to this end. Due to experimental limitations, very few of these data have been accumulated nnder simultaneous high pressure and temperature conditions. This has increased the uncertainty associated with, for example, estimates of lateral temperature variations from seismic tomography. Most current estimates use temperature coefficients of velocity based on laboratory data at ambient or near-ambient pressure.

Shock wave studies provide a means to simulate the ex-

Copyright 1992 by the American Geophysical Union.

Paper number 91JB02650.

0148-0227/92/91JB-02650\$05.00 treme pressures and temperatures of the Earth's interior. Density measurements under shock loading have played an important role in understanding the Earth. Recently, sound velocity measurements have been carried out under shock conditions for a number of metals, alloys, and minerals. The purpose of this paper is to evaluate the competing effects of temperature and pressure on sound velocities. The procedure will involve comparison of adiabatic extrapolations of ultrasonically measured sound velocities (infinitesimal strain) to Hugoniot measurements (finite strain) in order to constrain temperature coefficients of velocity as a function of pressure. The results will be compared to those from a variety of geophysical studies including seismic tomography, free oscillations, residual spheres, and the geoid. Duffy and Ahrens [1990] presented similar calculations in an abbreviated form.

\section{BACKGROUND AND THEORY}

\section{Sound Velocities From Shock Experiments}

Shock wave experiments are carried out by impacting a target with a high-speed flyer plate. The resulting shock propagates through the target, creating a state of compression. Typically, the impact velocity and the propagation speed of the shock front are measured experimentally. A review of experimental techniques for generating and recording shock states has been given by Ahrens [1987]. Conservation laws, known as the Rankine-Hugoniot conditions, are used to relate the kinematic flow variables to the thermodynamic variables (pressure, density, and internal energy) 
behind the shock front. A series of shock experiments generates a locus of points in pressure-volume-energy space known as the Hugoniot (Figure 1). Temperatures along the Hugoniot can be measured in some cases or calculated by making certain assumptions.

There has been long interest in measuring sound velocities behind the shock front in order to better constrain the thermodynamics of the shock state and to detect phase changes such as melting which are otherwise difficult to detect. Al'tshuler et al. [1960] conducted pioneering work on metals using lateral relaxation and overtaking techniques. Presently, there are two primary techniques for measuring sound speeds behind the shock front: the VISAR [Barker and Hollenbach, 1972] and the optical analyzer technique [McQueen et al., 1982]. VISAR is an acronym for velocity interferometer system for any reflector. In both methods, a thin flyer plate is used to impact the target (Figure 2). Upon impact, shock waves propagate both forward into the target and backward into the flyer. When the shock reaches the rear surface of the flyer, the pressure is released isentropically and a rarefaction fan propagates back through the flyer and the sample. The initial release propagates at the longitudinal sound velocity in a solid and the bulk velocity for a liquid. In solids, the bulk component of the release can also often be identified, although its interpretation may be more complicated because of wave interactions. Through a suitable experimental arrangement, the time required for rarefactions to propagate through the sample can be recorded. In the VISAR technique, the arrival of the shock and later release waves at a sample-window interface is monitored. Changes in particle velocity due to wave arrivals impart a Doppler shift to reflected laser light which is recorded in a modified Michelson interferometer. The VISAR has the advantage that a substantial portion of the release isentrope of the sample can be recovered [Chhabildas et al., 1988]. However, corrections must be made because of the impedance contrast between the sample and the window. These corrections are not required in the optical analyzer technique

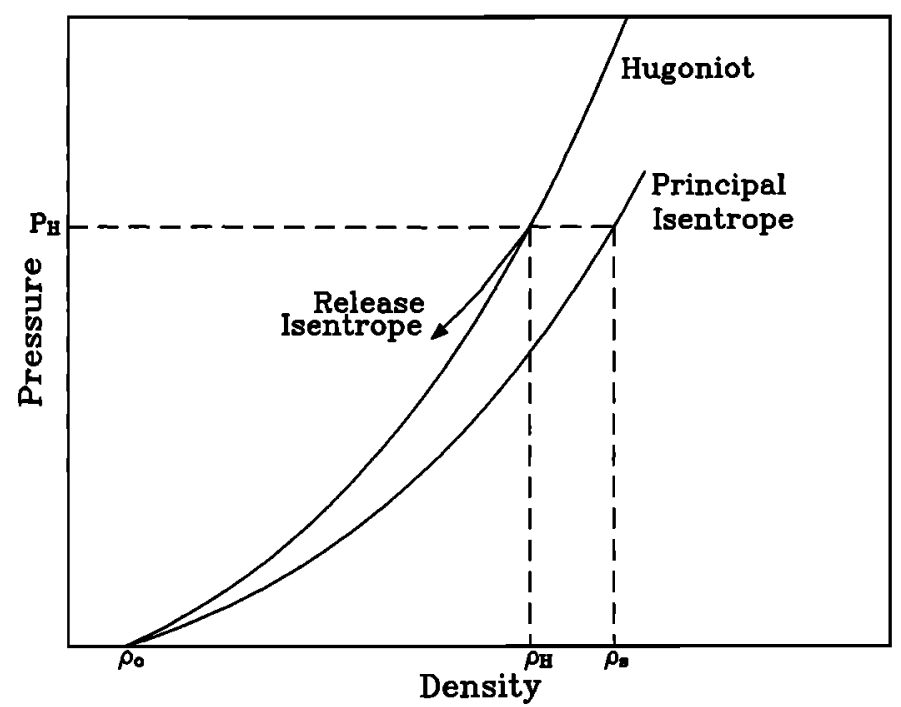

Fig. 1. Schematic pressure-density diagram illustrating relevant thermodynamic paths. $P$ - $\rho$ curves representing the Hugoniot and principal isentrope are shown for a material undergoing no phase transition. The bulk sound velocity in the shock state can be inferred from the initial slope of the release isentrope (equation (4)).

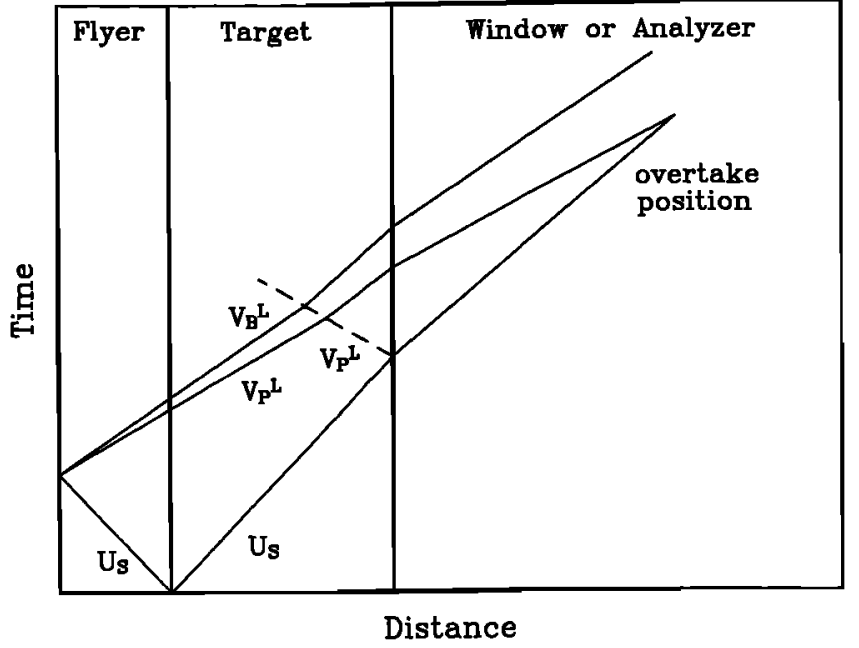

Fig. 2. Schematic Lagrangian distance-time diagram illustrating sound velocity measurements using the VISAR and optical analyzer techniques. Impact occurs at $t=0$, sending shocks into the flyer and target traveling with shock velocity $U_{S}$. Release waves are generated at the back surface of the flyer and propagate through the flyer and sample. Rarefactions traveling at the Lagrangian longitudinal and bulk sound velocities are indicated $\left(V_{P}^{L}\right.$ and $\left.V_{B}^{L}\right)$. The impedance contrast at the target/window interface produces a perturbation of the initial shock state which propagates through the target as shown by the dashed line. In the VISAR, wave arrivals at the target/window interface are monitored. In the optical analyzer technique, a series of overtake positions in the analyzer are measured using a stepped target.

which monitors the total thermal radiation emitted by an analyzer fluid in contact with the sample. The rarefaction propagates through the analyzer, eventually overtaking the shock. By simultaneously monitoring several positions on a stepped target, the effect of the analyzer can be eliminated and the sound velocity in the target determined. Typical uncertainties in sound velocities when measured with the optical analyzer technique are $1 \%$ while VISAR uncertainties are typically $2-3 \%$.

The Mie-Gruneisen equation is widely used in the analysis of shock data for minerals and metals:

$$
P-P_{H}=\rho \gamma\left(E-E_{H}\right)
$$

where $P$ is pressure, $E$ is energy, $\gamma$ is the Grüneisen parameter, and $\rho$ is the density. The subscript $H$ refers to Hugoniot conditions. This equation is useful in relating Hugoniot to off-Hugoniot states. The thermodynamic Grüneisen parameter is defined as

$$
\gamma \equiv \frac{\alpha K_{T}}{\rho C_{V}}
$$

where $\alpha$ is the thermal expansivity, $K_{T}$ is the isothermal bulk modulus, and $C_{V}$ is the constant volume specific heat. The Grüneisen parameter is often assumed to vary only with volume:

$$
\gamma=\gamma_{o}\left(\frac{\rho_{o}}{\rho}\right)^{q}
$$

where the subscript $o$ represents ambient-pressure conditions. Knowledge of the Hugoniot curve along with (1) and (3) allows determination of the initial slope of the release curve, thereby constraining the bulk sound velocity in the shock state (Figure 1)[McQueen et al., 1970; Ahrens, 1987]: 
$V_{B}=\sqrt{\frac{K_{S}}{\rho}}=\left\{\left(\frac{\partial P}{\partial \rho}\right)_{H}\left[1-\left(\frac{1}{\rho_{\mathrm{o}}}-\frac{1}{\rho}\right) \frac{\rho \gamma}{2}\right]+\frac{P_{H} \gamma}{2 \rho}\right\}^{\frac{1}{2}}$

where $V_{B}$ is the bulk sound velocity, $K_{S}$ is the adiabatic bulk modulus, and $(\partial P / \partial \rho)_{H}$ is the local slope of the Hugoniot. Bulk sound velocity measurements can be used to constrain $\gamma$ at high pressure through the use of (4). Available data, largely for fluid metals and alkali halides, are consistent with $q=1$ in (3) [Boness and Brown, 1990; Duffy and Ahrens, $1992 b$ ]. This is also supported by analyses of porous Hugoniot data [McQueen et al., 1970].

For many materials, an empirical relationship exists between the shock velocity, $U_{S}$, and the particle velocity, $u_{p}$ :

$$
U_{S}=c_{o}+s u_{p}
$$

where $c_{o}$ and $s$ are material constants. Density and pressure along the Hugoniot are then related according to:

$$
P_{H}=\frac{\rho_{o} \eta c_{o}^{2}}{(1-s \eta)^{2}}
$$

where

$$
\eta=\frac{u_{p}}{U_{S}}=1-\frac{\rho_{o}}{\rho}
$$

The measurement of shock temperatures has been an important new area of research in recent years [Ahrens, 1987]. In this work, however, we will largely rely on calculated temperatures. In the case where shock induced phase transitions do not occur, shock temperatures are obtainable from the differential equation [McQueen et al., 1970]:

$$
\rho \frac{d T_{H}}{d \rho}=\gamma T_{H}+\left[\frac{d P_{H}}{d \rho}\left(\frac{\rho}{\rho_{0}}-1\right)-\frac{P_{H}}{\rho}\right] / 2 C_{v}
$$

$T_{H}$ is the Hugoniot temperature. Electronic contributions to the specific heat can be important at high temperatures. Estimates of the electronic contributions are made following Shaner et al. [1984]. Temperatures along the principal isentrope can be obtained from the differential equation:

$$
\frac{d T}{T}=\gamma \frac{d \rho}{\rho}
$$

In calculating Hugoniot and isentrope temperatures, thermodynamic values of the Grüneisen parameter were used with $q=1$ in (3) [McQueen et al., 1970]. In order to relate pressures and volumes along the principal isentrope, we use finite strain theory together with parameters derived from nltrasonic experiments as discussed below.

\section{Finite Strain Theory}

Sound velocities along the principal isentrope will be used as a primary reference curve in this study. The principal isentrope is the isentrope originating at ambient pressure and temperature (Figure 1). The widely used finite strain equations of Birch [1938, 1952] express pressure, density, and the elastic moduli as functions of strain to large compressions. The expressions for the sound speeds have been presented to fourth order in several formulations [Sammis et al., 1970; Davies and Dziewonski, 1975; Jeanloz and Knittle, 19876. Pressure-volume data are frequently represented in the normalized pressure-normalized strain or $F$ - $f$ formulation [Birch, 1978]. The Eulerian strain, $f$, is defined as:

$$
f=\frac{1}{2}\left[\left(\frac{\rho}{\rho_{0}}\right)^{\frac{2}{3}}-1\right]
$$

The normalized pressure, $F$, is:

$$
F=\frac{P}{3 f(1+2 f)^{\frac{5}{2}}}
$$

In these coordinates, the Birch-Murnaghan equation to fourth order is:

$$
F=a_{0}+a_{1} f+a_{2} f^{2}
$$

where

$$
\begin{gathered}
a_{0}=K_{o S} \\
a_{1}=\frac{3 K_{o S}}{2}\left(K_{o S}^{\prime}-4\right) \\
a_{2}=\frac{3 K_{o S}}{2}\left[K_{o S} K_{o S}^{\prime \prime}+K_{o S}^{\prime}\left(K_{o S}^{\prime}-7\right)+\frac{143}{9}\right]
\end{gathered}
$$

where $K_{o S}$ is the zero-pressure adiabatic bulk modulus and single and double primes represent first and second pressure derivatives along the isentrope, respectively. This formulation has proven to be particularly convenient for the fitting of pressure-volume data since it reduces the non-linear Birch-Murnaghan equation to a simple quadratic in strain.

In an analogous manner, the sound velocities can be expressed as polynomials in strain through the use of normalized elastic moduli:

$$
\begin{aligned}
& M_{L}=\frac{C_{L}}{(1+2 f)^{\frac{5}{2}}}=\frac{\rho V_{P}^{2}}{(1+2 f)^{\frac{5}{2}}} \\
& M_{K}=\frac{K_{S}}{(1+2 f)^{\frac{5}{2}}}=\frac{\rho V_{B}^{2}}{(1+2 f)^{\frac{5}{2}}} \\
& M_{G}=\frac{G}{(1+2 f)^{\frac{5}{2}}}=\frac{\rho V_{S}^{2}}{(1+2 f)^{\frac{5}{2}}}
\end{aligned}
$$

where $M_{L}, M_{K}$, and $M_{G}$ are the normalized longitudinal, bulk, and shear moduli, respectively. $C_{L}$ is the longitudinal modulus and $\mathrm{G}$ is the shear modulus or rigidity. $V_{P}, V_{B}$, and $V_{S}$ are the compressional, bulk, and shear sound velocities. The longitudinal modulus, $C_{L}$ is given by:

$$
C_{L}=K_{S}+\frac{4}{3} G
$$

The finite strain expressions for the sound velocities to fourth order then become:

$$
\begin{gathered}
M_{L}=a_{L 0}+a_{L 1} f+a_{L 2} f^{2} \\
M_{K}=a_{K 0}+a_{K 1} f+a_{K 2} f^{2} \\
M_{G}=a_{G 0}+a_{G 1} f+a_{G 2} f^{2}
\end{gathered}
$$

The constants in the above expressions are given by the following:

$$
\begin{gathered}
a_{L 0}=C_{L o} \\
a_{L 1}=3 K_{o S} C_{L o}^{\prime}-5 C_{L o} \\
a_{L 2}=\frac{9}{2}\left[K_{o S}^{2}\left(C_{L o}^{\prime \prime}+\left(K_{o S}^{\prime}-4\right) \frac{C_{L o}^{\prime}}{K_{o S}}\right)+\frac{35 C_{L o}}{9}\right] \\
a_{K 0}=K_{o S} \\
a_{K 1}=3 K_{o S} K_{o S}^{\prime}-5 K_{o S}
\end{gathered}
$$




$$
\begin{gathered}
a_{K 2}=\frac{9}{2}\left[K_{o S}^{2}\left(K_{o S}^{\prime \prime}+\left(K_{o S}^{\prime}-4\right) \frac{K_{o S}^{\prime}}{K_{o S}}\right)+\frac{35 K_{o S}}{9}\right] \\
a_{G O}=G_{o} \\
a_{G 1}=3 K_{o S} G_{o}^{\prime}-5 G_{o} \\
a_{G 2}=\frac{9}{2}\left[K_{o S}^{2}\left(G_{o}^{\prime \prime}+\left(K_{o S}^{\prime}-4\right) \frac{G_{o}^{\prime}}{K_{o S}}\right)+\frac{35 G_{o}}{9}\right]
\end{gathered}
$$

The application of (10) and (20)-(31) allow zero-pressure elastic moduli and their pressure derivatives to be obtained from sound velocity or elastic constant measurements as a function of pressure.

\section{Results}

Hugoniot sound velocities have been reported for a number of metals, alloys, and minerals. We restrict attention to materials for which ultrasonic parameters determined near ambient pressure can be compared to Hugoniot measurements. In most cases, this means the material undergoes no polymorphic phase transitions along the Hugoniot. Bulk sound velocities in material shocked into the liquid regime will be considered separately.

In Figures 3-6, compressional and bulk sound velocities measured along the Hugoniot are shown for solid aluminum and aluminum alloys, copper, tungsten, tantalum, and the high-pressure phases of forsterite. Aluminum, copper, tungsten, and tantalum are materials that have been extensively studied under shock conditions and qualify as equation of state standards. The solid curves in each figure are fits to the Hugoniot data and the velocity near zero pressure. A convenient functional form to use is the following [Wang, 1988]:

$$
\ln V_{X}=d_{o}+d_{1} \ln P_{H}+d_{2} \ln ^{2} P_{H}
$$

where $X=P$ or $B$ for compressional or bulk velocities, respectively. The parameters of the fits to the Hugoniot data are given in Table 1 for pressure expressed in $\mathrm{GPa}$ and velocity expressed in $\mathrm{km} / \mathrm{s}$.

Aluminum is the most extensively studied material with both compressional and bulk sound speeds reported up to and beyond melting ( $\sim 150 \mathrm{GPa})$. The bulk sound velocities reported by Asay and Chhabildas [1981] are in poor agreement with the remaining data, probably due to wave interactions [Furnish, 1990]. For copper, tantalum, and the forsterite high-pressure phases (Figures 4-6) compressional sound velocities are reported up to the Hugoniot melting points. In the case of tungsten, both compressional and bulk sound speeds are reported up to $200 \mathrm{GPa}$ (Figure 5). Also shown in the figures are finite strain extrapolations ((20) and (21)) of the sound velocities for each material along its principal isentrope. The required coefficients (23)(28) are taken from low-pressure (typically $1 \mathrm{GPa}$ or less) high-precision ultrasonic measurements of moduli and their pressure derivatives listed in Table 2. Since second pressure derivatives cannot be reliably obtained from low-pressure data, the expressions were truncated at third order. That is, the last terms in (20) and (21) were taken to be zero. The small correction required to convert the measured pressure derivatives at constant temperature to constant entropy conditions is less than experimental error and has been ignored.

In Figure 6, the high-pressure phases of forsterite are taken to be an ideal equimolar mixture of periclase (MgO)

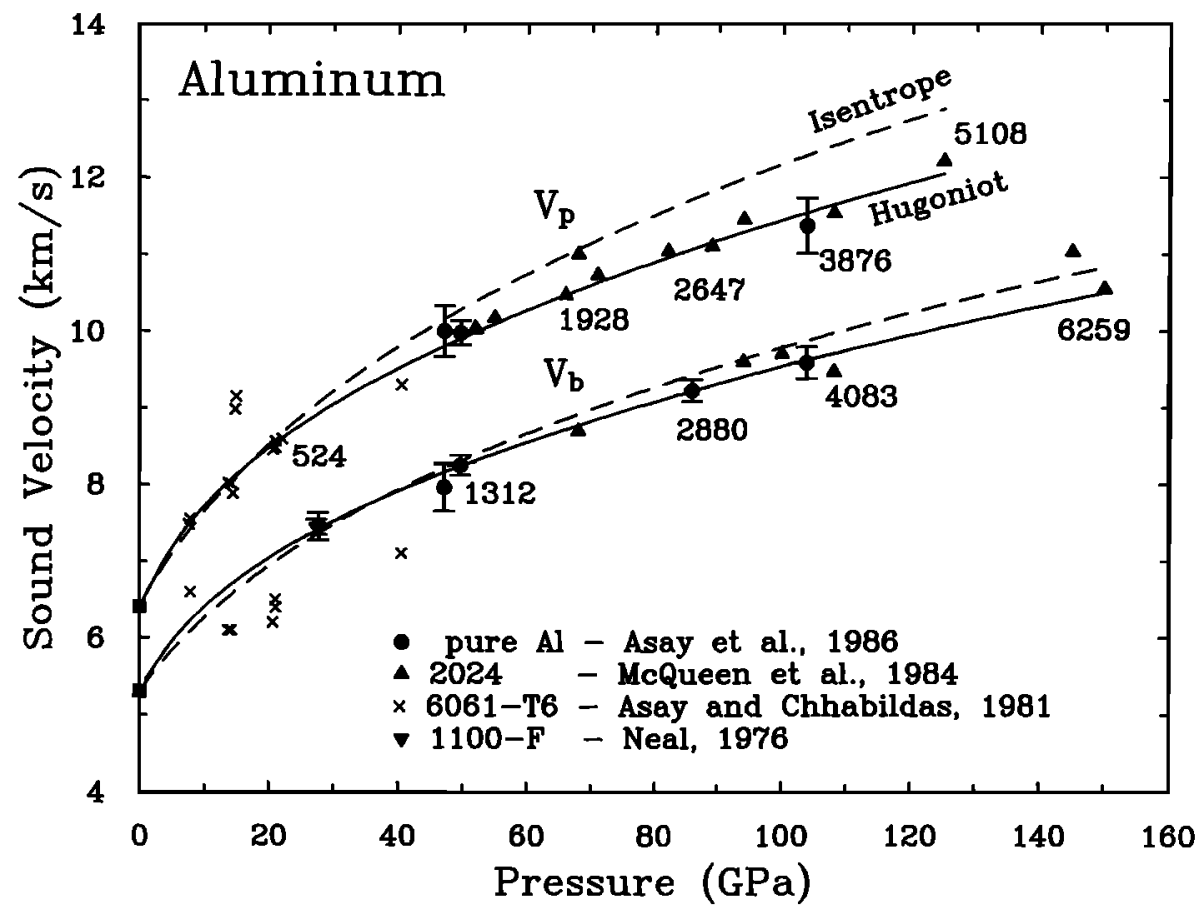

Fig. 3. Compressional and bulk sound velocities in aluminum and aluminum alloys along the Hugoniot. The Hugoniot melting point is near $150 \mathrm{GPa}$ [McQueen et al., 1984]. The solid curves are fits to the Hugoniot data (Table 1). The dashed curves are principal isentrope velocities obtained from third-order finite strain extrapolations of ultrasonic data (Table 2). Zero-pressure velocities are indicated by squares. The numbers near selected data points are the calculated shock temperatures in degrees Kelvin. 


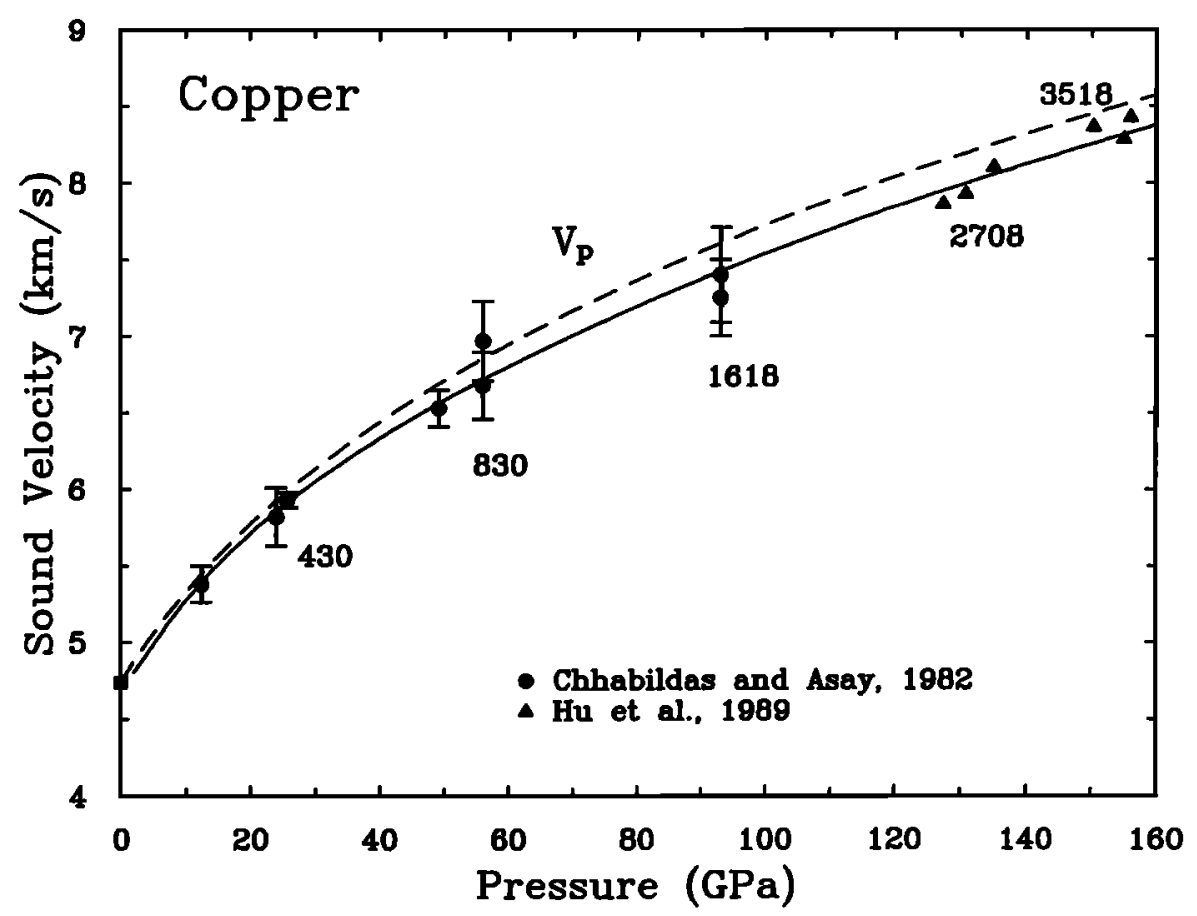

Fig. 4. Compressional sound velocities in copper along the Hugoniot. The Hugoniot melting point is near 170 GPa [Hu et al., 1989]. The solid curve is a fit to the Hugoniot velocities; the dashed curve represents the finite strain velocities along the isentrope. The numbers near selected data points are the calculated shock temperatures in degrees Kelvin.

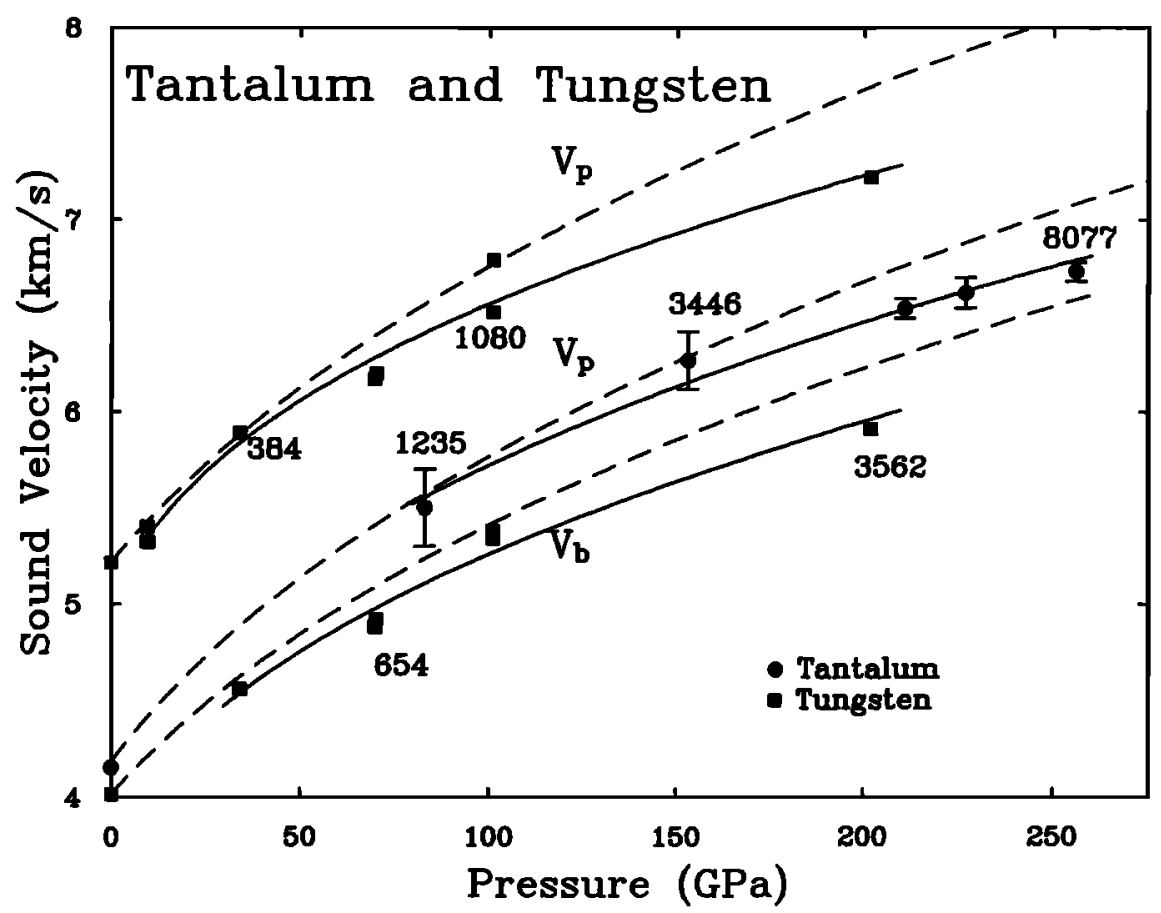

Fig. 5. Compressional sound velocities in tantalum (circles) and compressional and bulk sound velocities in tungsten (squares) along the Hugoniot. The Hugoniot melting point for Ta is near $295 \mathrm{GPa}$ [Brown and Shaner, 1984]. The solid curves are fits to the Hugoniot data; the dashed curves are finite strain extrapolations along the isentrope. The data for Ta are from Brown and Shaner [1984] and Asay et al. [1986], and for W from Asay et al. [1980] and Chkabildes et al. [1988]. The numbers near selected data points are the calculated shock temperatures in degrees Kelvin. 


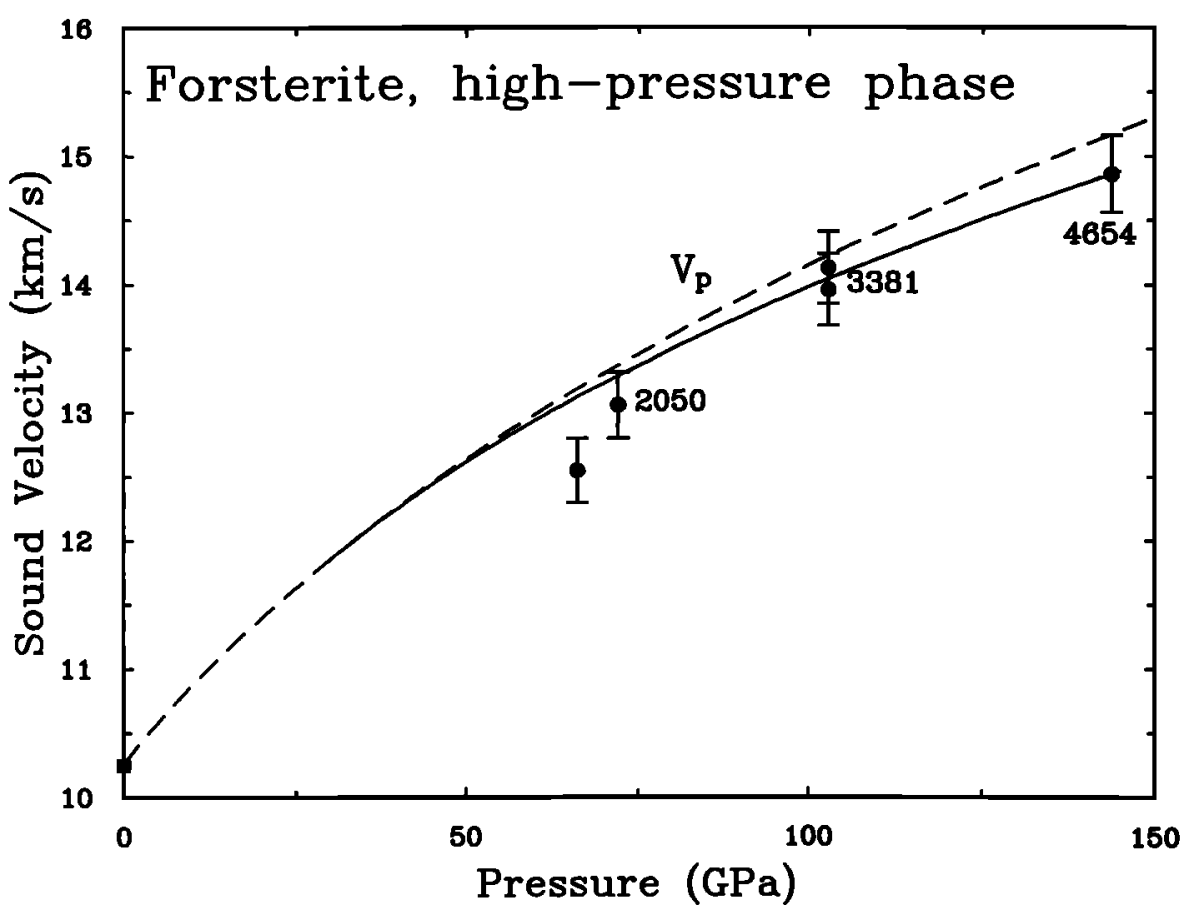

Fig. 6. Compressional sound velocities for the forsterite high-pressure phases along the Hugoniot. The Hugoniot melting point is near $150 \mathrm{GPa}$. The data points are from Brown et al. [1987]. The solid curve is a fit to the Hugoniot and zero-pressure data. The dashed curve is the finite strain trajectory for the forsterite high-pressure phases along the principal isentrope obtained from the properties of $\mathrm{MgO}$ and $\mathrm{MgSiO}_{3}$ (perovskite) as discussed in the text. The numbers near selected data points are the calculated shock temperatures in degrees Kelvin.

TABLE 1. Coeflicients of Poly nomial Fits to Hugoniot Sound Velocities

\begin{tabular}{lcrc}
\hline \multicolumn{1}{c}{ Material } & \multicolumn{1}{c}{$d_{0}$} & \multicolumn{1}{c}{$\begin{array}{c}d_{1}, \\
\times 10^{-2}\end{array}$} & \multicolumn{1}{c}{$\begin{array}{c}d_{2} \\
\times 10^{-2}\end{array}$} \\
\hline & $V_{P}$ & & \\
Aluminum & 1.8810 & 1.9252 & 2.2054 \\
Copper & 1.5705 & -1.7785 & 2.5057 \\
Tantalum & 1.4298 & -2.6315 & 2.0542 \\
Tungsten & 1.6565 & -3.0057 & 1.7130 \\
Forsterite & 2.3351 & -3.1485 & 2.1067 \\
high-pressure phase & & & \\
& $V_{B}$ & & \\
Aluminum & 1.6964 & 1.6984 & 2.2675 \\
Tungsten & 1.3959 & -4.7867 & 2.2840 \\
\hline
\end{tabular}

and perovskite $\left(\mathrm{MgSiO}_{3}\right)$. Wave velocities for the mixture were calculated by Voigt-Reuss-Hill averaging of the properties of the two components as a function of pressure. The parameters used in constructing the high-pressure isentrope are listed in Table 2. An additional uncertainty is that the pressure derivative of the shear modulus of perovskite is as yet unmeasured. We assume $d G / d P=2.0 \pm 0.5$ for this material, in accord with typical values for mantle minerals. Of the data in Figure 6, only those points above $100 \mathrm{GPa}$ are entirely in the high-pressure phase. The data below $75 \mathrm{GPa}$ are in the mixed phase region and contain some fraction of untransformed material.
TABLE 2. Elastic Parameters From Ultrasonic Experiments

\begin{tabular}{lrrrrrrc}
\hline Material & $\begin{array}{c}\rho_{o}, \\
\mathrm{~g} / \mathrm{cm}^{3}\end{array}$ & $\begin{array}{c}K_{o S}, \\
\mathrm{GPa}\end{array}$ & $\begin{array}{c}G_{o}, \\
\mathrm{GPa}\end{array}$ & $K_{o S}^{\prime}$ & $G_{o}^{\prime}$ & $\delta_{S}$ & Reference \\
\hline Aluminum & 2.70 & 76.0 & 26.1 & 5.11 & 2.04 & - & $a, b$ \\
& 2.70 & 75.9 & 26.1 & 4.41 & 1.82 & 3.0 & $c$ \\
Copper & 8.93 & 137.0 & 47.8 & 5.59 & 1.36 & 3.9 & $d$ \\
Tantalum & 16.68 & 195.7 & 72.1 & 3.77 & 0.97 & 4.0 & $d, e$ \\
Tungsten & 19.24 & 310.6 & 160.0 & 4.29 & 1.53 & 4.2 & $d, e$ \\
$\mathrm{MgSiO}_{3}, \mathrm{PV}$ & 4.11 & 246.4 & 184.2 & 3.9 & 2.0 & - & $f, g$ \\
$\mathrm{MgO}$ & 3.58 & 163.0 & 131.0 & 4.2 & 2.5 & 3.2 & $h, i$ \\
\hline
\end{tabular}

References: $a, H o$ and Ruoff [1969]; b, Schmunk and Smith [1959]; $c$, Thomas [1968]; $d$, Simmons and Wang [1971]; $e$, Katahara et al. [1979); $f$, Yeganeh-Haeri et al. [1989]; g, Knittle and Jeanloz [1987]; $h$, Sumino and Anderson [1984]; and $i$, Isaak et al. $[1989 a]$.

It can be seen from the figures that the Hugoniot measurements and finite strain extrapolations agree fairly well at low pressure but diverge increasingly as pressure increases. The procedure for analyzing these results will be first to compare the results at low pressure and then at high pressure where thermal effects are important.

\section{Evaluation of Finite Strain Parameters}

Studies at low pressure allow more or less direct comparison of ultrasonic extrapolations and Hugoniot sound velocities. Hugoniot sound velocities in aluminum alloys, cop- 
per, and tungsten at pressures below $35 \mathrm{GPa}$ are shown in Figure 7 along with finite strain extrapolations of ultrasonic data (Table 2). The temperatures achieved upon shocking to these pressures range between about 300 and $700 \mathrm{~K}$. Thermal effects on the sound velocities are therefore likely to be small. A maximum thermal correction can be estimated by using the zero-pressure temperature coeffcients from high-temperature ultrasonic experiments. The correction amounts to $3 \%$ or less; corrected velocities are indicated in the figure. Densities were corrected using the zero-pressure thermal expansion coefficients; the corrections are less than $1 \%$. The resulting pressure corrections were at most $5 \%$. For tungsten, the temperature correction is negligible. The aluminum Hugoniot data both here and in Figure 3 represent several alloys in addition to pure Al. Most alloys (such as 2024 and 6061) have Hugoniot properties and zero-pressure elastic moduli that are only slightly different from pure aluminum. Consequently, the ultrasonic data for aluminum are comparable to the alloy data. This conclusion is reinforced by the data of Figure 3 showing that sound velocities in 2024-Al and pure Al overlap in the 50-110 GPa pressure range.

There is reasonable agreement between the ultrasonic extrapolations in Figure 7 and the Hugoniot data. The temperature-corrected Hugoniot data were inverted using (20) to obtain elastic parameters for both the third and fourth-order finite strain formulations. The result are compared to ultrasonic parameters for aluminum and copper in Table 3. The zero-pressure velocities reported by Wang [1988] for both aluminum and copper are lower than ultrasonic values by $2-3 \%$. This may be due to initial porosity or in the case of aluminum, the type of alloy used. The extrapolated ultrasonic velocities agree with the temperature- corrected Hugoniot values within $1 \%$ when the ultrasonic extrapolations are shifted to correspond to the lower zeropressure velocities of Wang's samples (dashed curves in Figure 7). The aluminum 6061 data of Asay and Chhabildas [1981] fall within the range of the two finite strain extrapolations for aluminum. The two extrapolations differ by no more than $4 \%$ up to $150 \mathrm{GPa}$, and the actual extrapolation used does not affect the conclusions of this paper. Second pressure derivatives determined from fits to the shock data do not differ greatly from those resulting from the truncation of the finite strain expressions at third order. This indicates that higher order terms are not expected to significantly change the velocities at high pressure. The overall agreement between the finite strain velocities and Hugoniot measurements in this pressure range provide a reasonable basis for extrapolating the ultrasonic data to still higher pressures where thermal effects become important in the shock data.

Hugoniot and ultrasonic data may not be directly comparable due to a number of factors involved in the shockcompression process including material strength, thermodynamic disequilibrium, and shock-induced anisotropy or fabric [Grady, 1977]. For the metals under consideration here, material strength is not likely to be significant. Initial yield strengths are relatively small $(<1 \mathrm{GPa})$ for $\mathrm{Al}, \mathrm{Cu}, \mathrm{W}$, and Ta. While strain hardening does occur, results for aluminum indicate that material strength decreases above $\mathbf{5 0}$ GPa [Asay et al., 1986], possibly because of thermal effects. The comparison of Figure 7 suggests that Hugoniot sound velocities are not strongly affected by material strength. In addition, static isotherms and Hugoniot P-V curves based on the data of Tables 2 and 5 overlap in the low-pressure region. At higher pressures, strength effects are likely to be even less important because of the very high temperatures.

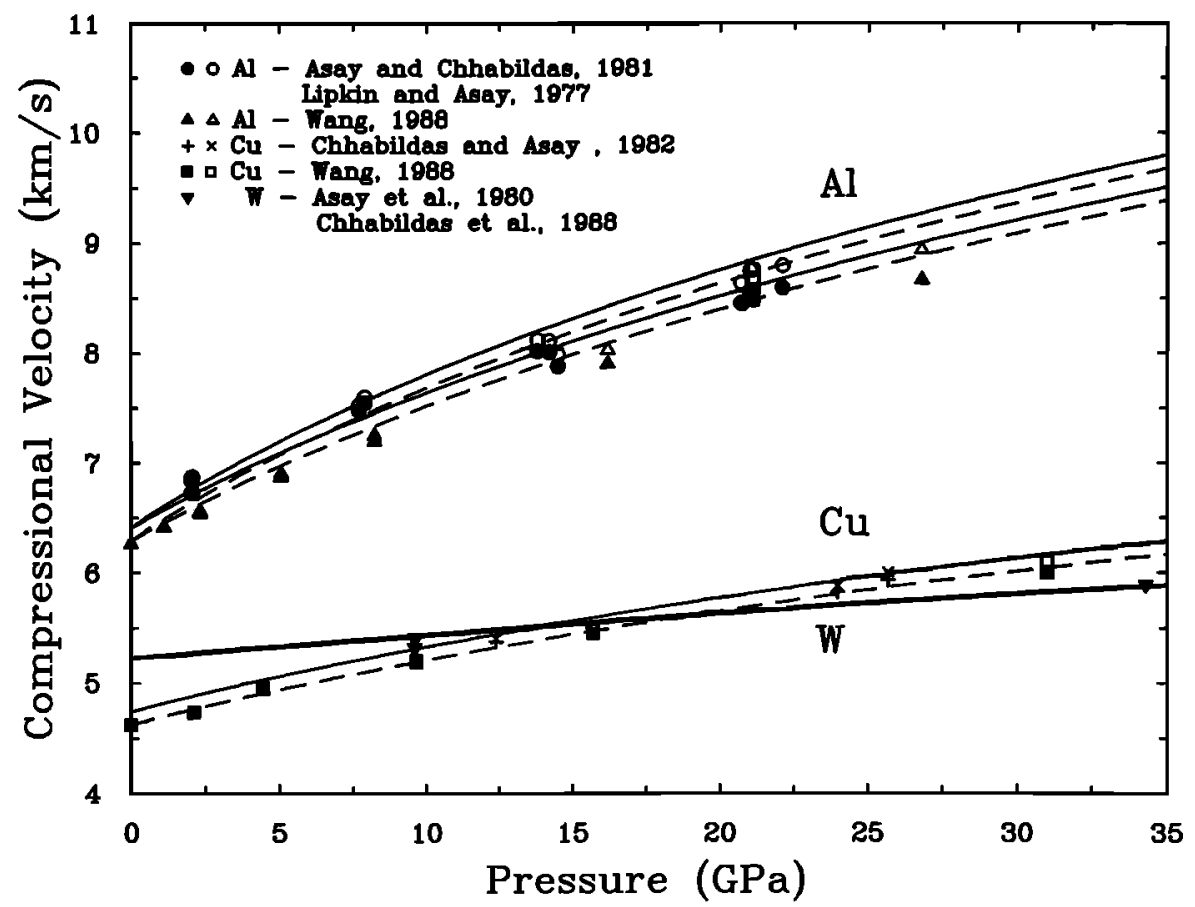

Fig. 7. Hugoniot sound velocity data below $35 \mathrm{GPa}$ for aluminum, copper, and tungsten. In the legend, symbols listed on the left correspond to Hugoniot velocities; those on the right represent velocities corrected to isentrope conditions using zero-pressure temperature coefficients. Solid curves are finite strain trajectories along the principal isentrope using the data of Table 2. The lower solid curve for aluminum corresponds to the data of Thomas [1968]. The dashed curves are finite strain trajectories shifted to correspond to the lower zero-pressure velocities of the data of Wang [1988]. 
TABLE 3. Compressional Elastic Properties From Ultrasonic and Hugoniot Data

\begin{tabular}{|c|c|c|c|}
\hline Study & $\begin{array}{l}C_{L_{0}} \\
\mathrm{GPa}_{2}\end{array}$ & $C_{L_{0}}^{\prime}$ & $\begin{array}{c}C_{L_{0}^{\prime}}^{\prime \prime} \\
\mathrm{GPa}^{-1}\end{array}$ \\
\hline \multicolumn{4}{|c|}{ Aluminum, Shock } \\
\hline \multicolumn{4}{|c|}{ Asay and Chhabildas [1981] } \\
\hline third order & 115.8 & 6.7 & - \\
\hline Fourth order & 115.8 & 6.7 & -0.11 \\
\hline \multicolumn{4}{|l|}{ Wang $|1988|$} \\
\hline third order & 109.0 & 7.1 & - \\
\hline fourth order & 110.8 & 6.2 & -0.01 \\
\hline \multicolumn{4}{|c|}{ Aluminum, Ultrasonics } \\
\hline Thomas [1968] & 110.8 & 6.8 & $(-0.11)$ \\
\hline Ho and Ruoff [1969] & 110.9 & 7.8 & $(-0.18)$ \\
\hline \multicolumn{4}{|c|}{ Copper, Shock } \\
\hline \multicolumn{4}{|c|}{ Chhabildas and Asay [1982] } \\
\hline third order & 199.7 & 7.2 & - \\
\hline fourth order & 200.7 & 6.5 & -0.01 \\
\hline \multicolumn{4}{|l|}{ Wang [1988] } \\
\hline third order & 190.7 & 7.3 & - \\
\hline Pourth order & 190.7 & 7.3 & -0.09 \\
\hline \multicolumn{4}{|c|}{ Copper, Vltrasonics } \\
\hline Simmons and Wang [1971] & 200.7 & 7.4 & $(-0.13)$ \\
\hline
\end{tabular}

It will also be shown below that sound velocities are also surprisingly high in shock-melted materials, for which material strength is not a factor. It is more difficult to evaluate strength effects for the high-pressure phases of forsterite. The initial yield strength of forsterite $(\sim 7 \mathrm{GPa})$ is larger than metal values. Brittle materials often exhibit a partial or complete loss of strength above the Hugoniot elastic limit however [Grady, 1977]. The high temperatures achieved in the high-pressure phase region may also significantly minimize strength effects.

\section{Temperature Coefficients at High Pressure}

Temperature derivatives of wave velocities at high pressure can be obtained from the data of Figures 3-6 by comparing velocities along the isentrope and the Hugoniot and by accounting for temperature differences between the curves. The expression for the magnitude of the temperature coefficients is:

$$
\left|\left(\frac{\partial V_{X}}{\partial T}\right)_{P}\right| \approx\left|\frac{V_{x H}-V_{x S}}{T_{H}-T_{S}}\right|
$$

where the subscripts $H$ and $S$ refer to Hugoniot and isentrope conditions. The resulting temperature derivatives are shown in Figures 8-12. Also shown in each figure is the zero-pressure temperature coefficient determined from hightemperature ultrasonic experiments. The error bars represent one standard deviation uncertainties which were determined through standard error propagation techniques including uncertainties in shock temperatures, measured velocities, and ultrasonic parameters.

The shock temperatures are computed as discussed previously (equation (8)). The Hugoniot parameters required for the temperature calculations are listed in Table 5. The uncertainties are substantial, largely because of uncertainties in the specific heat, but also because of uncertainties in the

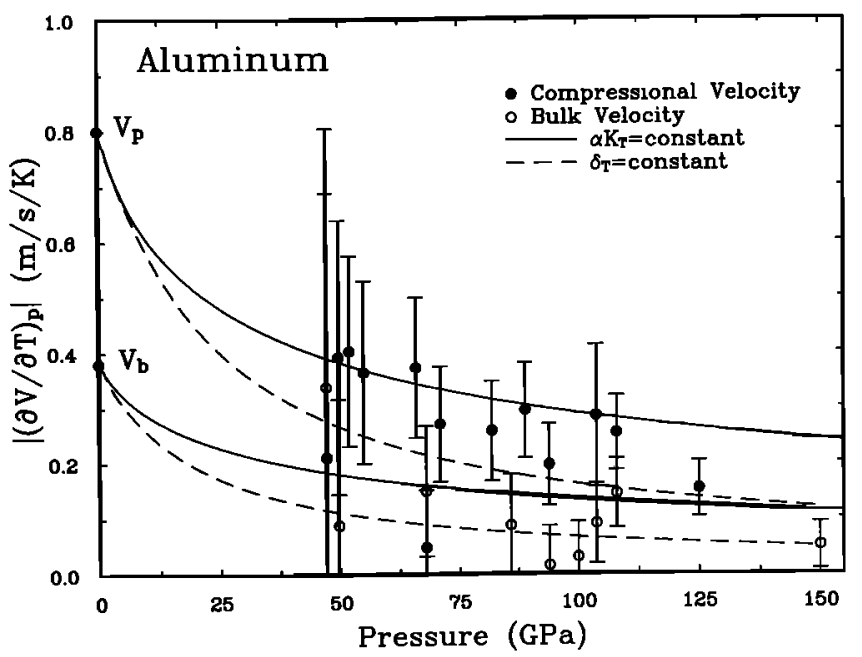

Fig. 8. Temperature derivatives of compressional and bulk sound velocities in aluminum and aluminum alloys as a function of pressure. Data of Figure 3 are shown for pressures greater than 45 GPa. Solid symbols are compressional velocity derivatives, open symbols are bulk velocity derivatives. Zero-pressure values are from Thomas [1968]. The dashed and solid lines are theoretical results based on (39) and (41), respectively.

Grüneisen parameter and its volume dependence (equations (2) and (3)). In the case of forsterite, measured temperatures between 153 and $175 \mathrm{GPa}$ [Lyzenga and Ahrens, 1980] provide additional constraints. While temperature uncertainties are large, the dominant contributor to the uncertainties in Figures 8-12 are the uncertainties in the velocities themselves. At low pressure, uncertainties in the Hugoniot and isentrope velocities are of the same order or greater than the differences between the two curves. Consequently, the temperature coefficients cannot be constrained very well. At higher pressures, where the separation between the isentrope and Hugoniot velocities is greater than experimental error, the temperature derivatives are better constrained.

An empirically based prediction of the magnitude of the temperature derivatives at high pressure is possible by using the dimensionless logarithmic anharmonic (DLA) parameters [Anderson, 1988]. The DLA parameter at constant pressure is:

$$
\{M\}_{P} \equiv\left(\frac{\partial \ln M}{\partial \ln \rho}\right)_{P}=-\frac{1}{\alpha M}\left(\frac{\partial M}{\partial T}\right)_{P}
$$

where $M$ is an elastic modulus $\left(K_{S}, K_{T}, \mathrm{G}, C_{L}\right)$ and $\alpha$ is the thermal expansion coefficient. It has been suggested that these parameters are relatively constant acros changes in crystal structure [Anderson, 1988]. They also appear to be relatively constant above the Debye temperature [Anderson and Goto, 1989; Isaak et al., 1989a,b]. The DLA parameters for the adiabatic $\left\{K_{S}\right\}_{P}$ and isothermal $\left\{K_{T}\right\}_{P}$ bulk moduli are referred to as $\delta_{S}$ and $\delta_{T}$, respectively. Recent high-pressure thermal expansion measurements [Chopelas and Boehler, 1989] suggest that $\delta_{T}$ is nearly constant along an isotherm to high pressure [Anderson et al., 1990]. If it is further assumed that $\delta_{T}$ as well as $\delta_{S}$ and $\{G\}_{P}$ are constant along the Hugoniot, then expressions for the temperature coefficients of velocities can be obtained as follows. Temperature derivatives of sound velocity are related to temperature derivatives of the elastic moduli according to: 


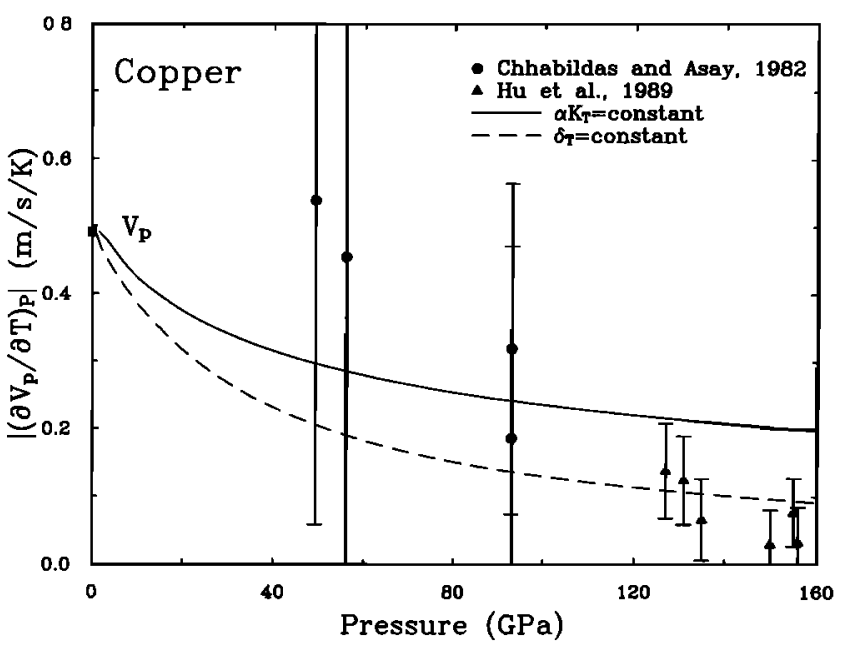

Fig. 9. Temperature derivatives of compressional velocity in copper above $40 \mathrm{GPa}$ using the data of Figure 4. The zero-pressure temperature coefficient is from Simmons and Wang [1971]. The dashed and solid lines are theoretical results based on (39) and (41), respectively.

$$
\dot{V}_{X} \equiv\left|\left(\frac{\partial V_{X}}{\partial T}\right)_{P}\right|=\left|\frac{V_{X}}{2}\left[\left(\frac{\partial \ln M}{\partial T}\right)_{P}+\alpha\right]\right|
$$

where $X=P, B$, or $S$ for compressional, bulk, and shear velocities, respectively, and $M$ is the corresponding elastic modulus $\left(C_{L}, K_{S}\right.$, or $\left.G\right)$. Pressure is expected to reduce the magnitude of the temperature derivatives of the moduli by suppressing anharmonicity [Zharkov and Kalinin, 1971; Anderson, 1987]. This implies that the mixed derivative $\left(\partial^{2} M / \partial P \partial T\right)$ should be greater than zero. Experimental constraints on $\left(\partial^{2} M / \partial P \partial T\right)$ are limited but most results do indicate that $\left(\partial^{2} M / \partial T \partial P\right)>0$ [Simmons and Wang, 1971]. A recent $a b$ initio model of the thermoelastic properties of $\mathrm{MgO}$ suggests $\left(\partial^{2} M / \partial T \partial P\right)>0$ for this material [Isaak et al., 1990]. Temperature derivatives of sound velocity slowly become more negative as temperature is increased [Anderson and Goto, 1989].

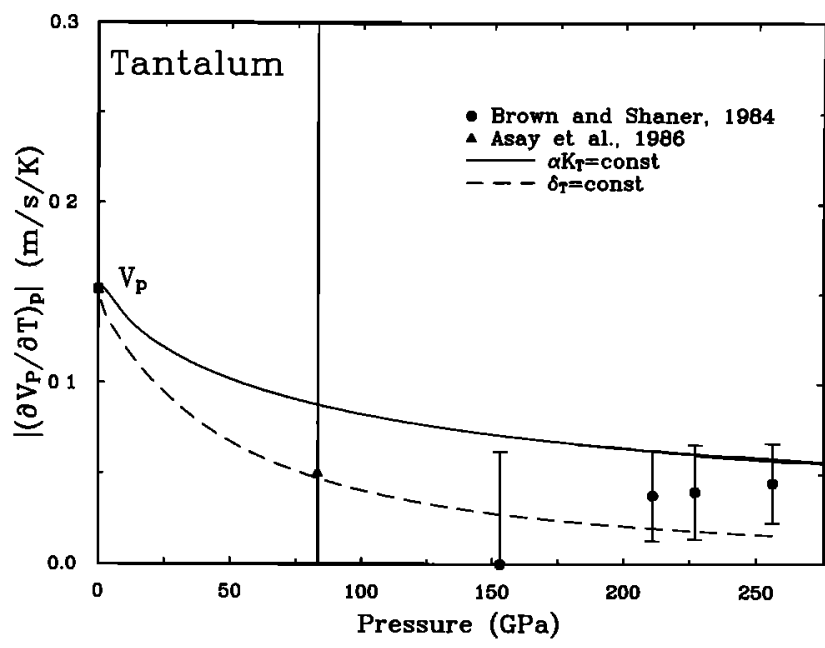

Fig. 10. Temperature derivatives of compressional velocity in tantalum using the data of Figure 5. The zero-pressure temperature coefficient is from Simmons and Wang [1971]. The dashed and solid lines are theoretical results based on (39) and (41), respectively.

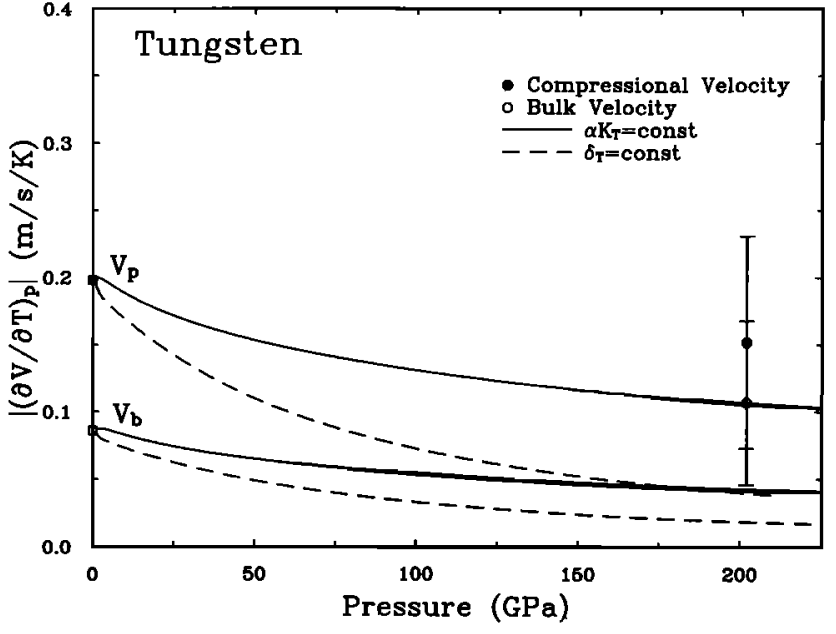

Fig. 11. Temperature derivatives of compressional and bulk sound velocities in tungsten for Hugoniot data of Figure 5 above $100 \mathrm{GPa}$. Solid symbols are compressional velocity derivatives, open symbols are bulk velocity derivatives. Zero-pressure values are from Simmons and Wang [1971]. The dashed and solid lines are theoretical results based on (39) and (41), respectively.

Combining expressions (34) and (35) yields:

$$
\dot{V}_{X}=\left|\frac{\alpha V_{X}}{2}\left[1-\{M\}_{P}\right]\right|
$$

For $\{M\}_{P} \approx$ constant, the temperature coefficient at pressure $P$ is related to the zero-pressure value by:

$$
\dot{V}_{X}=\dot{V}_{X \circ} \frac{\alpha V_{X}}{\alpha_{0} V_{x \circ}}
$$

Recent work on a range of materials is consistent with the following volume dependence for $\alpha$ [Anderson et al., 1990]:

$$
\left(\frac{\partial \ln \alpha}{\partial \ln \rho}\right)_{T}=-\delta_{T}
$$

which can be combined with (37) to give:

$$
\dot{V}_{X}=\dot{V}_{X \circ}\left(\frac{M}{M_{0}}\right)^{1 / 2}\left(\frac{\rho_{0}}{\rho}\right)^{\delta_{T}+1 / 2}
$$

Predicted temperature derivatives along the Hugoniot using (39) are compared to the experimental data in Figures 8-12. Values of $\delta_{T}$ were obtained from ultrasonic data by making use of the approximation [Anderson, 1987; Anderson and Goto, 1989]:

$$
\delta_{T} \approx \delta_{S}+\gamma
$$

The resulting $\delta_{T}$ values range between 5 and 6 for the materials studied here (Tables 2 and 5 ).

A second empirically based estimate of $\left(\partial V_{P} / \partial T\right)_{P}$ can be obtained from the definition of $\gamma$ and its volume dependence. Hugoniot sound velocity data are consistent with $\partial \ln \gamma / \partial \ln V \equiv q=1$ as discussed above. Together with (2), this implies that $\alpha K_{T} \approx$ constant along the Hugoniot, assuming $\partial \ln C_{P} / \partial \ln \rho \approx 0$ [Birch, 1968]. This relationship suggests a weaker dependence of $\alpha$ on density than implied by (38). Using values of $K_{S}$ along the Hugoniot from (4) or (32), the thermal expansivity along the Hugoniot can be estimated from:

$$
\alpha \approx\left[\frac{K_{S}}{\alpha_{o} K_{o T}}-\gamma T\right]^{-1}
$$




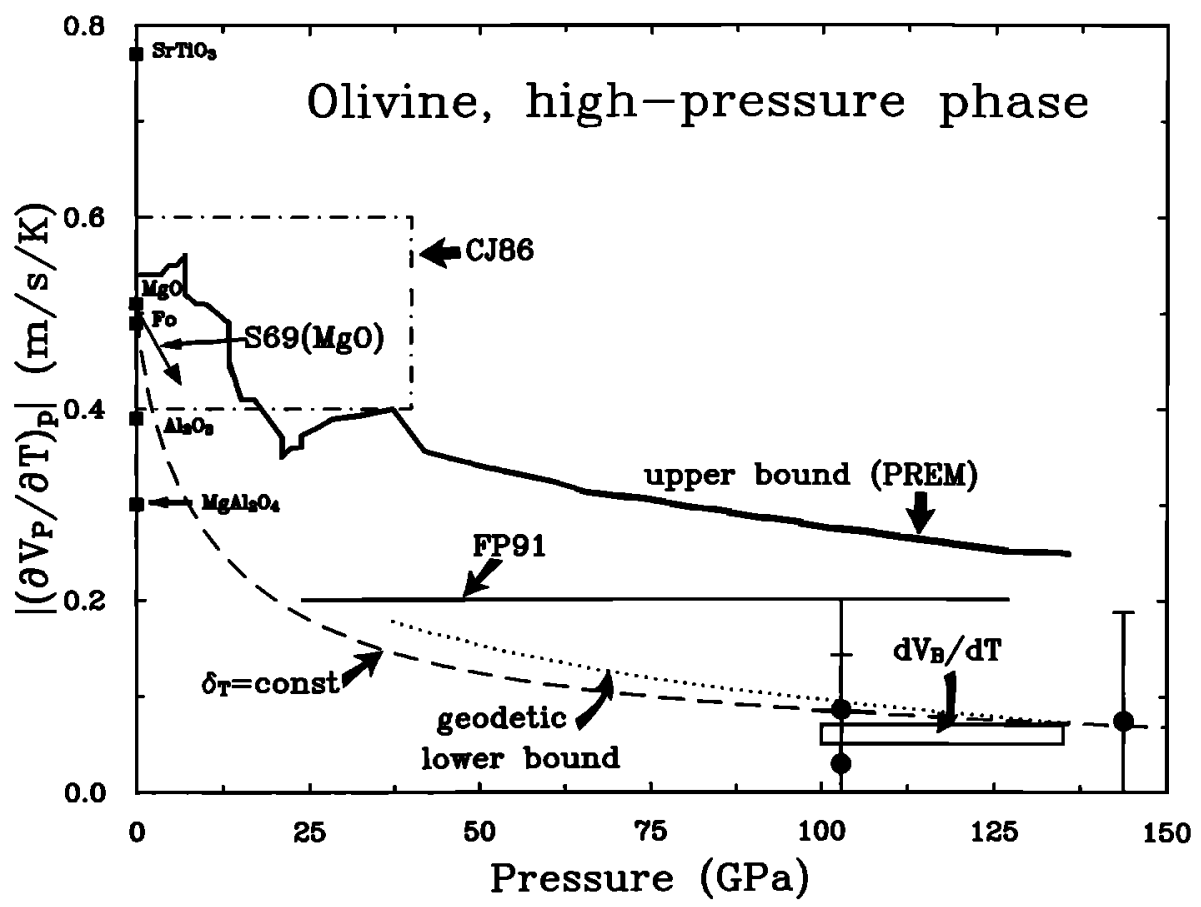

Fig. 12. Temperature derivative of compressional velocity for the forsterite high-pressure phase data of Figure 6. The high-pressure phase is assumed to be an ideal equimolar mixture of periclase and perovskite. Data below $75 \mathrm{GPa}$ are excluded because they are in the mixed phase region. The zero-pressure temperature coefficients for mantle-relevant minerals [Sumino and Anderson, 1984] are indicated by square symbols at zero pressure. The dash-dot box shows the range of temperature coefficients inferred by Creager and Jordan [1986] from their residualsphere study of slab anomalies. The arrow labeled $\mathrm{S} 69(\mathrm{MgO})$ represents an extension of the slope of $\left(\partial V_{P} / \partial T\right)_{P}$ determined from the ultrasonic data of Spetzler [1969] for $\mathrm{MgO}$. The thick solid line labeled upper bound (PREM) shows upper bound values for the magnitude of $\left(\partial V_{P} / \partial T\right)_{P}$ computed using thermoelastic properties for the Earth as discussed in the text. The dotted curve is a lower bound on the magnitude of $\left(\partial V_{P} / \partial T\right)_{P}$ inferred from geoid models. The line labeled FP91 shows values of $\left(\partial V_{P} / \partial T\right)_{P}$ inferred by Forte and Peltier [1991]. The box labeled $d V_{B} / d T$ shows the range of possible deep lower mantle values for this quantity based on (45). The dashed line represents predictions based on (39) using the $\delta_{T}$ of $\mathrm{MgO}$.

An estimate of the temperature coefficient of velocity can be made by inserting (41) in (35). If the temperature coefficients of the elastic moduli are assumed constant with pressure, this should provide an upper bound on $\left|\left(\partial V_{P} / \partial T\right)_{P}\right|$. The temperature coefficients of the elastic moduli are expected to decrease in magnitude with pressure because of reduced anharmonicity, but the magnitude of the decrease is uncertain. Upper bound estimates of $\left|\left(\partial V_{P} / \partial T\right)_{P}\right|$ based on (41) are shown in Figures 8-12. In Figure 12, the upper bound has been constructed by combining thermoelastic properties of the Earth with temperature coefficients of elastic moduli of mantle minerals. This is further discussed in the next section. It is clear from Figures 8-12 that (41) does bound most of the data. Above 1 Mbar, the decrease in the magnitude of the temperature coefficients of the elastic moduli required by the data for $\mathrm{Al}, \mathrm{Cu}, \mathrm{Ta}$, and $\mathrm{Mg}_{2} \mathrm{SiO}_{4}$ when $\alpha$ is given by (41) ranges from a factor of 1 to 4 .

The temperature derivative of $K_{S}$ is related to the pressure dependence of $\alpha$ through the following relationship:

$$
\left(\frac{\partial K_{S}}{\partial T}\right)_{P}=K_{T}\left[K_{S}\left(\frac{\partial \alpha}{\partial P}\right)_{T}+\alpha \gamma+\gamma T\left(\frac{\partial \alpha}{\partial T}\right)_{P}\right]
$$

where the temperature dependence of $\gamma$ has been neglected. At high $P$ and $T$, the temperature dependence of $\alpha$ also becomes small [Isaak et al., 1990] and the third term on the right can be neglected. Neglect of $(\partial \alpha / \partial T)_{P}$ at low $P$ and $T$ will overestimate $\left|\left(\partial K_{S} / \partial T\right)_{P}\right|$. A particular pressure dependence for $\alpha$ (e.g., (38) or (41)) therefore implies a particular temperature dependence of $K_{S}$. Model calculations for $\mathrm{MgO}$ along a $2000 \mathrm{~K}$ isotherm show that $\left|\left(\partial K_{S} / \partial T\right)_{P}\right|$ decreases by about $40 \%$ from $0.023 \mathrm{GPa} / \mathrm{K}$ to $0.013 \mathrm{GPa} / \mathrm{K}$ from $P=0$ to $P=135 \mathrm{GPa}$ when $\alpha$ is described by either (38) or (41). This supports the arguments above for the pressure decrease of $\left|\left(\partial K_{S} / \partial T\right)_{P}\right|$. It is also consistent with estimates of $\left|\left(\partial K_{S} / \partial T\right)_{P}\right|$ from the deep lower mantle using seismological data as discussed in the next section. Thermodynamic considerations provide no insight on the $T$ dependence of the shear modulus, however. Therefore, we have continued to assume the temperature derivatives are constant with pressure to obtain the upper bound estimates shown in Figures 8-12.

For aluminum, the temperature derivatives of both compressional and bulk velocities decrease with pressure, with a factor of 3-8 decrease in the absolute values of the temperature derivatives at the highest pressures (Figure 8 ). If the ultrasonic data of Ho and Ruoff [1969] and Schmunk and Smith [1959] is used rather than the data of Thomas [1968], the temperature derivatives at high pressure are increased by about $50 \%$ and agreement with (38) and (41) is poor. However, the low-pressure Hugoniot data appears to be in better agreement with the data of Thomas [1968](Figure 7). For copper and tantalum, the decrease in the temperature coefficients of the compressional velocities are also large, declining by factors of about 6 and 3, respectively. Only for tungsten, 
the most incompressible of the metals studied, are the temperature derivatives at high pressure indistinguishable from low-pressure values within their uncertainties. The results for the forsterite high-pressure phases are broadly similar to the more compressible metals. For the three data points above $100 \mathrm{GPa}$, the temperature coefficient of $V_{P}$ has an average value of $-0.1 \pm 0.1 \mathrm{~m} / \mathrm{s} / \mathrm{K}$ compared with $-0.5 \mathrm{~m} / \mathrm{s} / \mathrm{K}$ for forsterite and $\mathrm{MgO}$ under ambient conditions [Sumino and Anderson, 1984; Isaak et al., 1989a,b].

In Table 4, mixed pressure-temperature derivatives of compressional velocity are obtained from linear fits to the data in Figures 8-12. The derivatives are positive, except for tungsten for which the derivatives are not resolvably different from zero. The incompressible metals, tungsten and tantalum, have mixed derivatives about an order of magnitude smaller than the more compressible metals and forsterite. For the high-pressure phases of forsterite, the zero-pressure mixed derivative was obtained from a least squares fit using high-pressure temperature coefficients and zero-pressure values from ultrasonic data for forsterite and $\mathrm{MgO} . \partial^{2} V_{P} / \partial P \partial T$ is inversely proportional to the longitudinal modulus, $C_{L}$, for the materials studied here (Figure 13).

Using expressions (5)-(7) and the data of Table 5, Hugoniot pressures can be related to Hugoniot densities. Following a similar procedure to that used above, temperature coefficients at constant density, $\left(\partial V_{X} / \partial T\right)_{\rho}$, can be obtained. The results differ from the constant pressure case in that

TABLE 4. Mixed Pressure-Temperature Velocity Derivatives

\begin{tabular}{lccc}
\hline Material & $\begin{array}{l}C_{L_{a}}, \\
\mathrm{GPa}\end{array}$ & $\begin{array}{r}\partial^{2} V_{P} / \partial P \partial T, \\
10^{-0}(\mathrm{~km} / \mathrm{s}) / \mathrm{GPaK}\end{array}$ & $\begin{array}{c}\partial^{2} V_{B} / \partial P \partial T, \\
10^{-0}(\mathrm{~km} / \mathrm{s}) / \mathrm{GPaK}\end{array}$ \\
\hline Aluminum & 111 & 6.1 & 2.6 \\
Copper & 201 & 3.6 & - \\
Forsterite & 237 & 3.3 & - \\
Tantalum & 292 & 0.4 & - \\
Tungsten & 524 & 0.2 & -0.1 \\
\hline
\end{tabular}

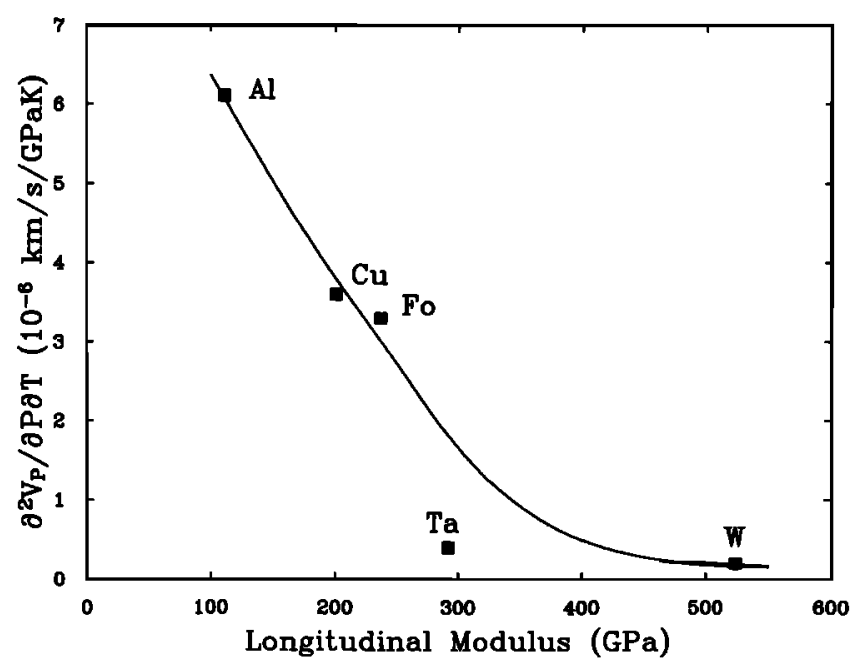

Fig. 13. The pressure derivative of $\left|\left(\partial V_{P} / \partial T\right)_{P}\right|$ plotted as a function of zero-pressure longitudinal modulus. Pressure derivatives were obtained by fitting zero-pressure ultrasonic temperature derivatives and high-pressure shock values from Figures 8-12. velocity differences between the isentrope and the Hugoniot are somewhat smaller in magnitude and can be either positive or negative.

The results of this section show that temperature coeffcients of velocity can be obtained from Hugoniot sound velocity measurements on solids at high pressure. Most metals and the high-pressure phases of forsterite exhibit marked reduction in the magnitude of the temperature derivatives at high pressure. Thus, the effect of temperature on sound velocities at high pressure is considerably less than would be predicted on the basis of zero-pressure sound velocity data. The experimentally determined temperature derivatives also agree well with the prediction of (39) except in the case of tungsten (Figures 8-12). This suggests that the assumption $\{M\}_{P} \approx$ const holds reasonably well at high pressure and temperature. Alternatively, assuming $(\partial M / \partial T)_{P}$ and $\alpha K_{T}$ are constant provides an upper bound for the magnitude of the temperature coefficient of velocity, although tungsten is again an exception. These results illustrate the tradeoff between $(\partial M / \partial T)_{P}$ and $\alpha$ in (35).

\section{Bulk Velocities in Liquids}

In addition to the solid data discussed above, there is a large body of Hugoniot sound velocity data on metals and minerals that have undergone shock melting. In Figures 14 and 15, these data are shown for alkali halides and metals. Also shown in each figure are third-order finite strain extrapolations of the bulk sound velocity for each material in the solid phase (Table 6). These plots exhibit a number of differences from those involving shocked solids. In many cases, the Hugoniot velocities for the liquids are as fast or faster than the extrapolated velocities for the solid phase. This is surprising in view of the extremely high temperatures achieved in these experiments. For the alkali halides, the temperatures between 50 and $150 \mathrm{GPa}$ have been measured to be in the 5000-15,000 K range [Boness and Brown, 1990] and a substantial temperature effect might be expected. Furthermore, the tendency for the isentrope and Hugoniot velocities to diverge at high pressure is much reduced and of the opposite sign for the liquid data. Another surprising feature is that in nearly all cases, the finite strain extrapolations based on the properties of the solid are within a few per cent of the measured Hugoniot velocities for the liquid.

There are several possible explanations for these features. The temperature coefficients of velocity for liquid metals and alkali halides may be very small. Another possibility is that the first or second pressure derivatives for the liquid

TABLE 5. Hugoniot Equation of State Parameters

\begin{tabular}{lrccccc}
\hline Material & $\begin{array}{c}\rho_{o}, \\
\mathrm{~g} / \mathrm{cm}^{3}\end{array}$ & $\begin{array}{c}c_{o}, \\
\mathrm{~km} / \mathrm{s}\end{array}$ & 8 & $\gamma_{0}$ & $\begin{array}{c}C_{V}, \\
\mathrm{~J} / \mathrm{gK}\end{array}$ & Reference \\
\hline Aluminum & 2.70 & 5.386 & 1.339 & 2.00 & 0.902 & $a, b$ \\
Copper & 8.93 & 3.940 & 1.489 & 1.99 & 0.372 & $b$ \\
Tantalum & 16.68 & 3.414 & 1.200 & 1.64 & 0.137 & $b, c$ \\
Tungsten & 19.24 & 4.029 & 1.237 & 1.65 & 0.131 & $b, c$ \\
Forsterite & 3.20 & 3.80 & 1.69 & 2.0 & - & $d$ \\
\hline
\end{tabular}

References: a, Asay et al. [1986]; b, McQueen et al. [1970]; c, Kalahara et al. [1979|; and d, Brown el al. [1987]. 


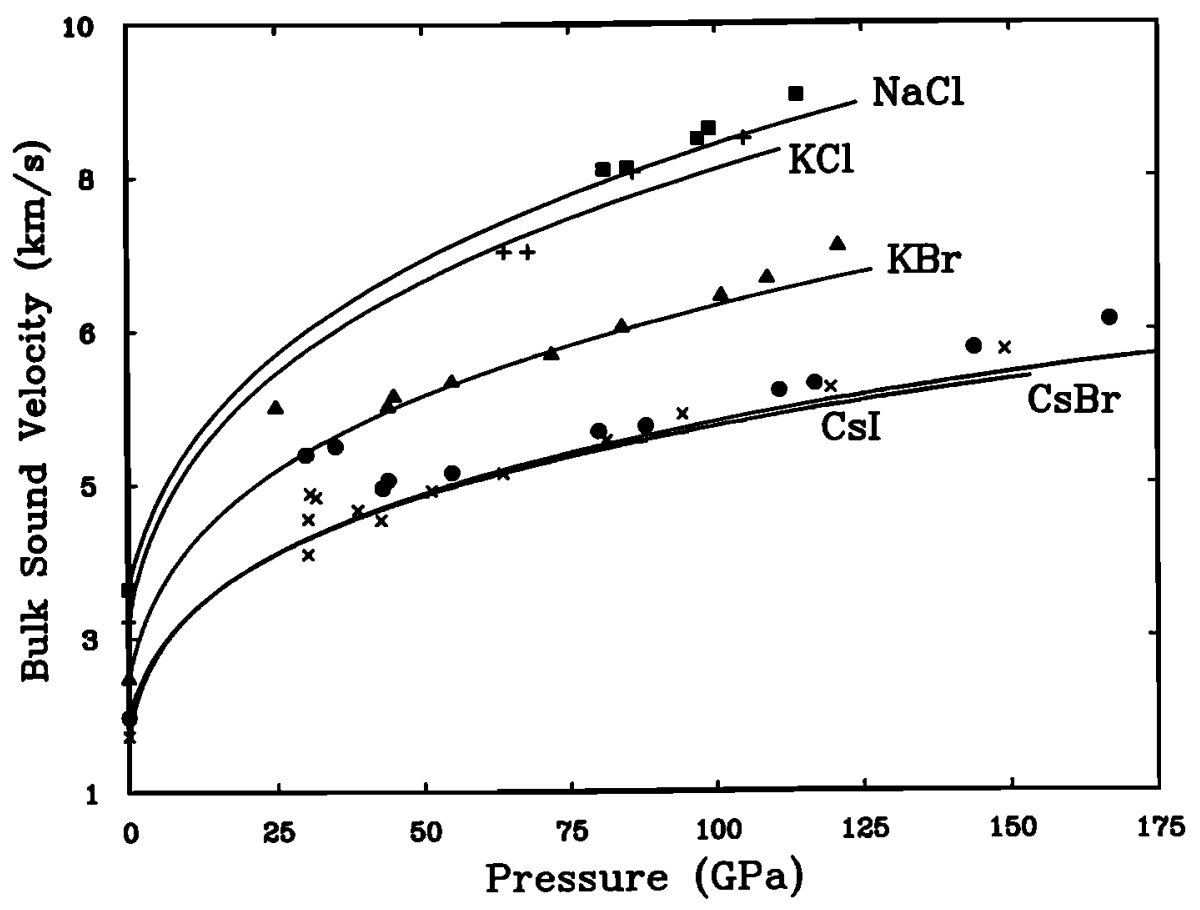

Fig. 14. Bulk sound velocities in shock-melted alkali halides. The curves are third-order finite strain extrapolations based on ultrasonic properties of the corresponding solid (Table 6). Unlike the solids, the Hugoniot velocities are similar to or slightly faster than the finite strain extrapolations despite the large temperature differences between the curves. The Hugoniot data are from Swenson et al. [1986] and Boness and Brown [1990].

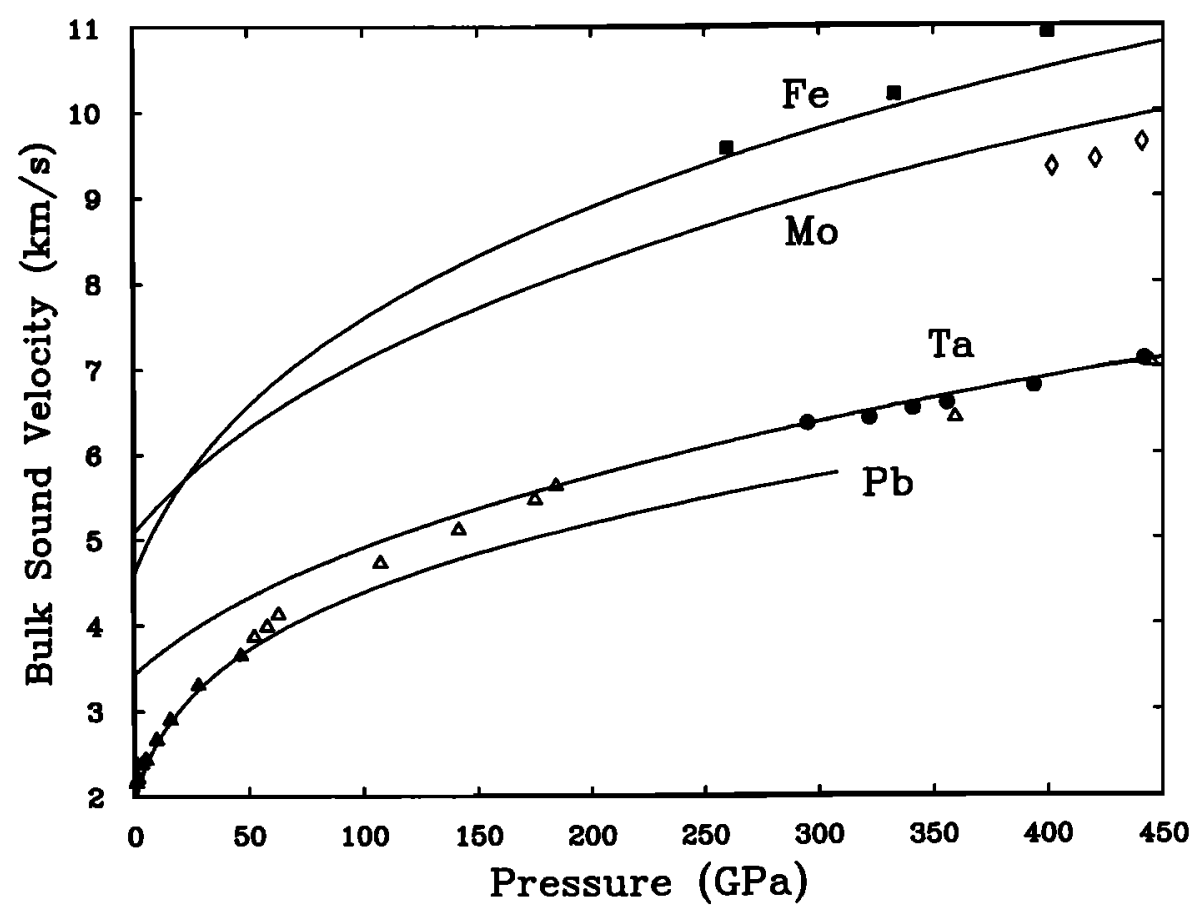

Fig. 15. Bulk sound velocities in shock-melted metals. The curves are third-order finite strain extrapolations based on ultrasonic properties of the corresponding solid (Table 6). With the exception of lead above $100 \mathrm{GPa}$, the Hugoniot sound velocities are close to finite strain predictions despite large temperature differences between the curves. The Hugoniot data are from Brown and Shaner [1984], Brown and McQueen [1986], Boness et al. [1988], Wang [1988], and Hixson et al. [1989]. 
TABLE 6. Elastic Parameters for Alkali Halides and Metals

\begin{tabular}{cccc}
\hline Material & $\begin{array}{c}\rho_{o}, \\
\mathrm{~g} / \mathrm{cm}^{3}\end{array}$ & $\begin{array}{c}K_{o S}, \\
\mathrm{GPa}\end{array}$ & $K_{o S}^{\prime}$ \\
\hline \multicolumn{4}{c}{ Alkali Halides } \\
$\mathrm{KCl}$ & 1.99 & 18.3 & 5.36 \\
$\mathrm{NaCl}$ & 2.16 & 25.2 & 5.26 \\
$\mathrm{KBr}$ & 2.75 & 14.9 & 5.38 \\
$\mathrm{CsBr}$ & 4.45 & 15.8 & 5.38 \\
$\mathrm{CsI}$ & 4.53 & 12.5 & 7.13 \\
& Metals & \\
$\mathrm{Fe}$ & 7.87 & 166.0 & 5.29 \\
$\mathrm{Mo}$ & 10.21 & 263.0 & 4.40 \\
$\mathrm{~Pb}$ & 11.34 & 44.7 & 5.53 \\
$\mathrm{Ta}$ & 16.68 & 195.7 & 3.77
\end{tabular}

Alkali halide data from Sumino and Anderson [1984]; metal data from Simmons and Wang [1971].

may be larger than is appropriate for the solid. The effect of changes in physical properties (such as $\rho$ and $K_{S}$ ) across the melt boundary may also play a role. There are insufficient data to compare finite strain extrapolations based on liquid properties with the Hugoniot data. For all the materials studied, except perhaps for lead above $100 \mathrm{GPa}$, bulk velocities in the liquid along the Hugoniot are predicted quite well to first order by the ultrasonic parameters for the solid with no temperature correction. It has also been noted that Birch's law (velocity linear with density) is approximately satisfied for shocked fluid alkali halides and metals [Shaner et al., 1988; Boness and Brown, 1990], as well as for solid metals [Duffy and Ahrens, 1992a].

\section{GEOPHYSICAL IMPLICATIONS}

Pressure Dependence of Temperature Coefficients of $V_{P}$ and $V_{B}$

Seismological data have been used to estimate temperature coefficients of velocity in the Earth in a number of studies. Sleep [1973] obtained the result $\left|\left(\partial V_{P} / \partial T\right)_{P}\right|=0.9$ $\mathrm{m} / \mathrm{s} / \mathrm{K}$ from a study of subduction in the Aleutians. More recently, McNutt and Judge [1990] constrained the temperature coefficient of the shear velocity to $125 \mathrm{~km}$ depth to be $-0.618 \mathrm{~m} / \mathrm{s} / \mathrm{K}$ based on the variation of Love wave velocity with lithospheric age. Both these results are about a factor of two larger than typical laboratory values for silicates (Figure 12). Residual sphere studies use travel time residuals from subduction zone earthquakes to constrain the geometry and depth extent of slabs. This addresses the question of whether slabs penetrate into the lower mantle. The temperature coefficient of velocity is an important scaling parameter in these studies, relating the observed anomalies to the thermal structure of the slab. The results of these studies are consistent with $\partial V_{P} / \partial T=-0.5 \pm 0.1 \mathrm{~m} / \mathrm{s} / \mathrm{K}$ over a depth range from about 150 to $1000 \mathrm{~km}$ [Creager and Jordan, 1986; Jordan et al., 1989; Fischer et al., 1991; Boyd and Creager, 1991]. Furthermore, the results of residual sphere studies imply $\partial V_{P} / \partial T$ is a weak function of depth and does not vary significantly between the upper and lower mantle [Jordan et al., 1989]. The shock wave data discussed above cannot be directly compared to the residual sphere results, as the shock data lies at higher pressure. Nevertheless, the data are inconsistent in that the shock results require a substantial decrease in the magnitude of the temperature coefficients with pressure. The residual sphere results are consistent with the upper bound values of $\left|\left(\partial V_{P} / \partial T\right)_{P}\right|$ inferred in Figure 12 and these studies may not be sensitive to relatively small changes in $\partial V_{P} / \partial T$ over restricted pressure ranges. Fischer et al. [1988] considered the tradeoff between depth extent of the slab and $\partial V_{P} / \partial T$. It was found that decreasing the magnitude of $\partial V_{P} / \partial T$ increases the required penetration depth of the slab. Other studies, however, suggest that the residual sphere velocity anomalies may be overestimated due to deep mantle and near-receiver effects [Zhou et al., 1990] and that slab velocity heterogeneity may be relatively weak at depths greater than $300 \mathrm{~km}$ [Schwartz et al., 1991].

The present results may be more directly applied to the deep lower mantle $(P>100 \mathrm{GPa})$. The temperature coefficient of velocity within this region is important in relating seismically observed velocity heterogeneities to the underlying thermal anomalies. A number of recent studies have converted velocity anomalies to density anomalies which, when incorporated into dynamic Earth models, can be used to predict the observed geoid [Hager et al., 1985; Forte and Peltier, 1987; Ricard et al., 1989; Hager and Richards, 1989; Hager and Clayton, 1989]. The parameter relating velocity and density anomalies, $\left(\partial V_{P} / \partial \rho\right)_{P}$, inferred from these studies is model dependent and values between 2 and $10(\mathrm{~km} / \mathrm{s}) /\left(\mathrm{g} / \mathrm{cm}^{3}\right)$ have been reported. The results depend strongly on the assumed radial viscosity structure and lateral variations in viscosity may be important as well [Richards and Hager, 1989]. When combined with the thermal expansion coefficient, an estimate of $\left(\partial V_{P} / \partial T\right)_{P}$ can be made from these results. Given the range of possible values of $\alpha$ and $\left(\partial V_{P} / \partial \rho\right)_{P}$ in the lower mantle, $\left(\partial V_{P} / \partial T\right)_{P}$ is only weakly constrained. A lower bound for this quantity from the geodetic and seismic data is indicated in Figure 12. With the present work, an independent estimate of $\left(\partial V_{P} / \partial \rho\right)_{P}$ based solely on mineral physics data may be constructed. Using $\left(\partial V_{P} / \partial T\right)_{P}=-0.1 \pm 0.1 \mathrm{~m} / \mathrm{s} / \mathrm{K}$ for $P>100 \mathrm{GPa}$, together with $\alpha=10 \pm 5 \times 10^{-6} K^{-1}$, we find $\left(\partial V_{P} / \partial \rho\right)_{P}$ $=2(\mathrm{~km} / \mathrm{s}) /\left(\mathrm{g} / \mathrm{cm}^{3}\right)$ with acceptable values ranging from 0 to 8 . This overlaps most of the range of $\left(\partial V_{P} / \partial \rho\right)_{P}$ from geodetic data discussed above.

An important area of uncertainty involves the value of the thermal expansivity under deep mantle conditions. The values used above $\left(\alpha=5-15 \times 10^{-6} K^{-1}\right)$ cover the range of estimates for the mantle and mantle minerals at pressure above 1 Mbar based on both data and thermodynamic calculations [Stacey, 1977; Anderson, 1987; Anderson et al., 1990]. The value of $\alpha$ has important implications for the dynamics and composition of the lower mantle [Chopelas and Boehler, 1989]. The assumption $\alpha K_{T}=$ constant discussed previously implies the higher value $\left(\alpha \approx 15 \times 10^{-6} \mathrm{~K}^{-1}\right)$ is appropriate for the deep lower mantle, while if $\delta_{T}=$ const, the lower value $\left(\alpha \approx 5 \times 10^{-6} \mathrm{~K}^{-1}\right)$ is more appropriate. A value of $40 \times 10^{-6} \mathrm{~K}^{-1}$ has been used as the ambientpressure thermal expansion coefficient of lower mantle material [Knittle et al., 1986]. The tradeoff between $\alpha$ and $(\partial M / \partial T)_{P}$ in (35) precludes the use of the present data to distinguish between the thermal expansivity models.

For a given pressure dependence of $\alpha$, an upper bound for the magnitude of $\left(\partial V_{P} / \partial T\right)_{P}$ in the Earth can be cal- 
culated using (35) and PREM parameters [Dziewonski and Anderson, 1981] for the mantle. This is illustrated in Figure 12, using the thermal expansivities of Stacey [1977] and assuming an upper bound for $\left|\left(\partial C_{L} / \partial T\right)_{P}\right|$ of $0.036 \mathrm{GPa} / \mathrm{K}$ in the upper mantle and $0.048 \mathrm{GPa} / \mathrm{K}$ in the lower mantle. These are ambient-pressure values for forsterite and $\mathrm{MgO}$, respectively. Varying $\alpha$ within the bounds established by the two relations discussed above changes the upper bound by $\pm 10 \%$. These results then establish an upper bound of $\left|\left(\partial V_{P} / \partial T\right)_{P}\right|=0.28 \mathrm{~m} / \mathrm{s} / \mathrm{K}$ for $P$ above $1 \mathrm{Mbar}$ in the lower mantle. The present results for the forsterite highpressure phase between 103 and $144 \mathrm{GPa}$ can be satisfied by approximately a $50 \%$ reduction in $\left|\left(\partial C_{L} / \partial T\right)_{P}\right|$ relative to ambient-pressure values for $\mathrm{MgO}$.

The temperature derivative of the bulk velocity, $\left(\partial V_{B} / \partial T\right)_{P}$, in the mantle can be estimated from thermodynamic and seismological parameters for the Earth. Stacey and Loper [1983] have shown that, under the assumption $\left(\partial K_{T} / \partial T\right)_{V} \approx 0$, the temperature coefficient of the bulk modulus can be approximated by:

$$
\left(\frac{\partial K_{S}}{\partial T}\right)_{P} \approx \alpha K_{S}\left[\frac{\alpha K_{S}}{\rho C_{P}}-\left(\frac{\partial K_{S}}{\partial P}\right)_{S}\right]
$$

This expression can aso be obtained by substituting $q=1$ into the thermodynamic relationship of Bassett et al. [1968] and neglecting the difference between the adiabatic and isothermal moduli and pressure derivatives

$$
q=1-(1+\alpha \gamma T) \delta_{S}-\left(\frac{\partial K_{S}}{\partial P}\right)_{T}+\gamma
$$

Combining (35) and (43) yields the following expression:

$$
\left(\frac{\partial V_{B}}{\partial T}\right)_{P} \approx \frac{\alpha V_{B}}{2}\left[\frac{\alpha K_{S}}{\rho C_{P}}-\left(\frac{\partial K_{S}}{\partial P}\right)_{S}+1\right]
$$

Stacey and Loper [1983] present a similar equation (their equation (54)) for $\left(\partial V_{P} / \partial T\right)_{P}$ which incorrectly neglects the temperature dependence of the shear modulus. Using $\alpha=$ $5-15 \times 10^{-6} \mathrm{~K}^{-1}$ and $C_{P}=1.26 \mathrm{~J} / \mathrm{gK}$ [Stacey, 1977] in (45) results in $\left|\left(\partial V_{B} / \partial T\right)_{P}\right| \approx 0.05-0.07 \mathrm{~m} / \mathrm{s} / \mathrm{K}$ between 100 and $135 \mathrm{GPa}$, a threefold to fourfold reduction relative to the zero-pressure value for $\mathrm{MgO}$ of $\left|\left(\partial V_{B} / \partial T\right)_{P}\right|=0.21 \mathrm{~m} / \mathrm{s} / \mathrm{K}$ [Sumino and Anderson, 1984] (Figure 12). Thus, thermodynamic estimates of the temperature coefficient of the bulk velocity for the bottom portion of the lower mantle yield results similar to $\left(\partial V_{P} / \partial T\right)_{P}$, both in magnitude and in total reduction relative to zero-pressure laboratory values. Estimates of $\left(\partial K_{S} / \partial T\right)_{P}$ from (43) range from -0.008 to -0.015 $\mathrm{GPa} / \mathrm{K}$ at $100 \mathrm{GPa}$, while laboratory values for mantle minerals range from -0.014 to $-0.021 \mathrm{GPa} / \mathrm{K}$, suggesting this parameter may decrease in magnitude by a factor of up to 2.5 across the mantle. This is consistent with our results and with the experimental data and theoretical studies discussed above which suggest that pressure reduces the magnitude of the temperature coefficients of elastic moduli.

Forte and Peltier [1991] employed viscous flow models to estimate $\left(\partial V_{P} / \partial T\right)_{P}$ in the lower mantle from seismically observed core-mantle boundary topography. Using Stacey and Loper's value for $\left(\partial V_{P} / \partial T\right)_{P}$ discussed above in the $D^{\prime \prime}$ region, they fit the magnitude of the CMB topography using $\left(\partial V_{P} / \partial T\right)_{P}=-0.2 \mathrm{~m} / \mathrm{s} / \mathrm{K}$ for the remainder of the lower mantle (Figure 12). In an ultrasonic study, Spetzler [1969] measured sound velocities in $\mathrm{MgO}$ to $1 \mathrm{GPa}$ and 800 $K$. Figure 12 shows the extension of the $\left(\partial V_{P} / \partial T\right)_{P}$ slope defined by this data. These results also suggest a marked reduction in the magnitude of $\left(\partial V_{P} / \partial T\right)_{P}$ with pressure.

\section{Interpretation of Relative Variations of $P$ and $S$ Wave Ve- locity}

Tomographic maps of lower mantle $P$ and $S$ wave velocity variations are correlated but relative shear wave anomalies are larger than $P$ wave anomalies. This can be expressed for the lower mantle in the form [Dziewonski and Woodhouse, 1987; Davies, 1990]:

$$
\nu=\left(\frac{\partial \ln V_{S}}{\partial \ln V_{P}}\right)_{P}=2.0 \text { to } 2.5
$$

Comparison of different lower mantle models may introduce error due to noise or differences in model parameterization. However, similar results have been obtained from the Earth's free oscillation spectra which constrain $\nu$ to lie between 1.67 and 2.56 with $75 \%$ confidence, with an optimum value of 2.3. These data exclude the zero-pressure value of 1.25 at the $96 \%$ confidence interval [ $L i$ et al., 1991]. For mantle minerals near ambient conditions, $\nu=0.7$ to 1.4 (Figure 16). At ambient pressure, $\nu$ does not increase at temperatures up to $1700 \mathrm{~K}$ for $\mathrm{MgO}$ and $\mathrm{Mg}_{2} \mathrm{SiO}_{4}$ [Isaak et al., 1989a,b]. Anderson [1987] divided the temperature derivatives of the elastic moduli into intrinsic (anharmonic) and extrinsic (quasiharmonic) components in an attempt to determine the separate effects of pressure and temperature on the moduli. It was proposed that increasing pressure decreases the effect of temperature on moduli partly because of greater suppression of the extrinsic (volume-dependent) temperature effect. The intrinsic temperature derivative of the bulk modulus is generally smaller than the intrinsic derivative of the rigidity. Consequently temperature variations should affect the rigidity more than the bulk modulus at elevated pressure. Attempts to verify this idea using potential models have reached conflicting conclusions [Reynard and Price, 1990; Agnon and Bukowinski, 1990]. Reynard and Price [1990] obtain values of $\delta_{T}$ for several mantle minerals that are in good agreement with the experimental data of Chopelas and Boehler [1989] and exhibit only a weak pressure dependence. Agnon and Bukowinski [1990], on the other hand, find that $\delta_{S}$ decreases strongly with pressure for oxides. The decrease in $\delta_{S}$ appears to be sufficient to allow $\nu \geq 2$ in the lower mantle. The results of Reynard and Price [1990] suggest that large $\nu$ values in the lower mantle cannot be explained solely by temperature variations of velocity.

For aluminum and tungsten, compressional and bulk wave velocities can be combined to constrain the shear velocity to within $\sim 5 \%$ along the Hugoniot [Duffy and Ahrens, 1992a]. Comparing these to isentrope velocities allows logarithmic velocity variations to be constrained (Figure 16). The aluminum results are based on the fits of Table 1 since there are only a few pressures at which both $V_{P}$ and $V_{B}$ are reported. Results at lower pressures than shown exhibit a great deal of scatter and are not considered reliable because the difference in velocity between the isentrope and Hugoniot is small. Figure 16 shows that $\nu$ values at high pressure are similar to zero-pressure values for both $\mathrm{Al}$ and $\mathrm{W}$. While uncertainties are considerable, the large increases in $\nu$ of the type required to explain the seismological result are not required by the shock wave data. More data, particularly on geologically relevant materials, is needed to confirm this result. Other possible explanations for large $\nu$ values in the 


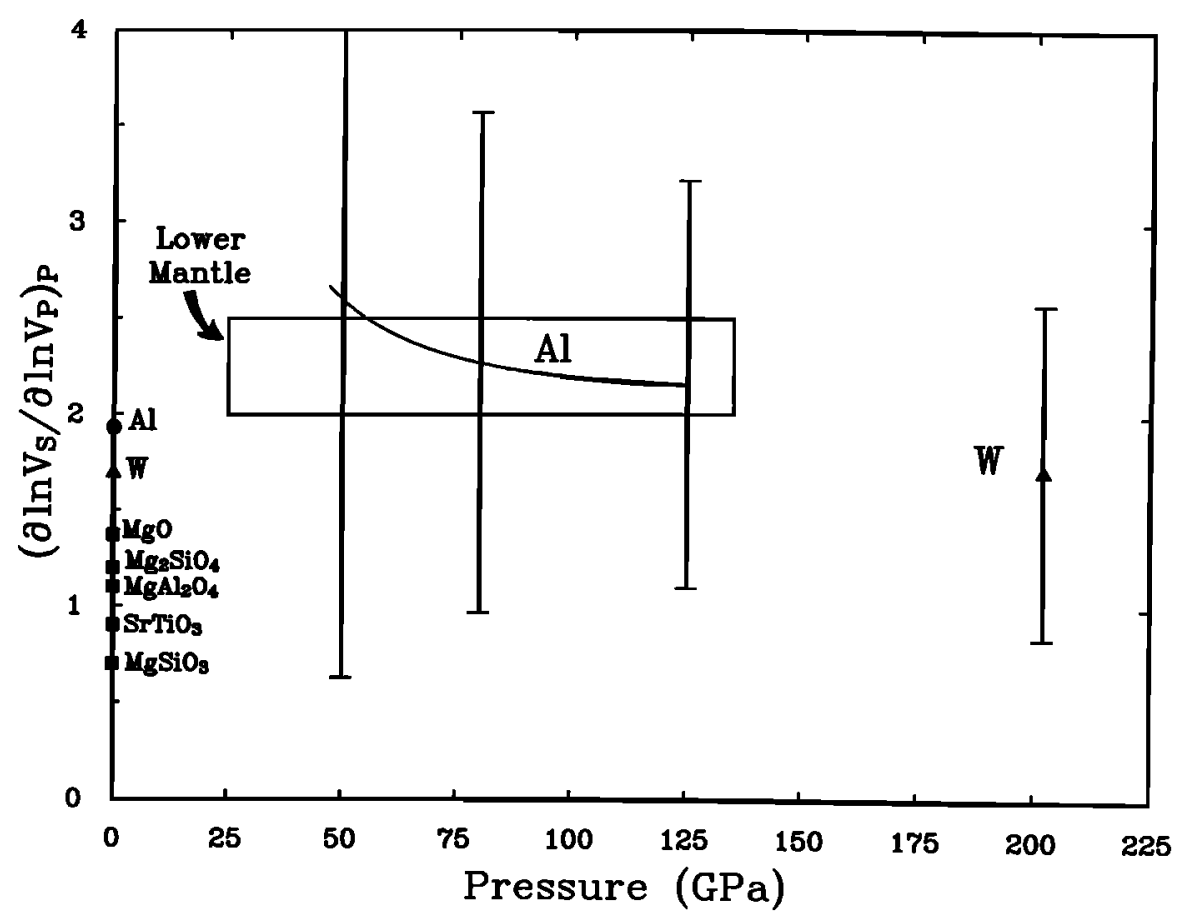

Fig. 16. The relative variation of shear to compressional wave velocity, $\nu=\left(\partial \ln V_{S} / \partial \ln V_{P}\right)_{P}$, plotted as a function of pressure for aluminum and tungsten. Seismic tomography and free oscillation spectra require that $\nu$ be significantly greater in the lower mantle (box) than zero-pressure values for mantle minerals. Zero-pressure values for aluminum and tungsten are also shown.

lower mantle include the presence of small amounts of tluid, partial melting [Duffy and Ahrens, 1992b] and a ferroelastic phase transition in perovskite [Yeganeh-Haeri et al., 1989].

\section{Thermal Variations in the Lower Mantle}

Tomographically determined seismic velocity variations are often interpreted as thermal anomalies related to mantle convection. Throughout most of the lower mantle, the RMS amplitude of $P$ wave anomalies is approximately $0.1 \%$ [Clayton and Comer, 1983; Dziewonski, 1984; Gudmundsson et al., 1990]. Assuming these anomalies are entirely due to temperature and using a zero-pressure temperature coefficient for scaling, the magnitude of long-wavelength thermal anomalies in the lower mantle has been estimated to be 12-27 $\mathrm{K}$ [Hager and Clayton, 1989; Machetel, 1990]. A corresponding analysis for $S$ waves yields anomalies of $70 \mathrm{~K}$ [Davies, 1990]. The shock data presented here, together with empirically based predictions and geophysical data, suggest that $\left|\left(\partial V_{P} / \partial T\right)_{P}\right|$ is reduced by a factor of $2-8$ relative to zeropressure values at pressures of $100 \mathrm{GPa}$ or more. Thermal anomalies in the deep lower mantle are therefore estimated to be $120 \pm 100 \mathrm{~K}$. The anomalies in the $\mathrm{D}^{\prime \prime}$ region must be a factor of 3-4 larger than this to satisfy seismic data. The degree to which tomography provides an accurate representation of mantle anomalies is an area of active research [Machetel, 1990; Su and Dziewonski, 1991]. We have assumed that seismic velocity variations are directly the result of large-scale temperature anomalies in the mantle.

In a stochastic analysis of global $P$ wave travel time data, Gudmundsson et al. [1990] determined the standard deviation of long-wavelength slowness variations as a function of depth. Standard deviations are approximately $0.5 \%$ at the top of the upper mantle, $0.3 \%$ in the transition region, and $0.1 \%$ in the lower mantle. The smaller variations in the lower mantle could be explained by the depth variation of $\left|\left(\partial V_{P} / \partial T\right)_{P}\right|$ inferred in Figure 12. The stochastic model also exhibits jumps in slowness deviations near the surface, the $670-\mathrm{km}$ discontinuity, and the mantle-core boundary. This may reflect the effect of thermal boundary layers or possibly sampling problems in these regions. The observed magnitude of long-wavelength slowness variations could be explained by long-wavelength thermal anomalies of near constant magnitude throughout the mantle, except near thermal boundary layers, and the behavior of $\left|\left(\partial V_{P} / \partial T\right)_{P}\right|$ indicated in Figure 12. Changes in the scale length of the anomalies and changes in the amount of small scale power between the upper and lower mantle, as well as near boundary layers, have also been identified by Gudmundsson et al. [1990].

As a final point, application of the present shock wave results to the Earth's interior must be done with cantion as most data presently available are for metals rather than silicates. Since the properties of these metals are well characterized by shock studies and they do not undergo solid-solid phase transitions in the region studied, they represent the best data set to infer the general behavior of sound velocities at high pressure and temperature. Furthermore, more limited data for silicates suggest they behave analogously, although interpretation is less certain because of the effects of phase transitions. It has also been demonstrated that the silicate data is consistent with theoretical expectations and with geophysical data.

\section{SUMMARY}

Temperature coefficients of sound velocity are important in relating seismically observed velocity anomalies to their 
underlying thermal causes. Compressional and bulk sound velocity measurements on shocked solids show that temperature derivatives of velocity decrease strongly at high pressure. This is based on a comparison of Hugoniot velocities with third-order Eulerian finite strain extrapolations of ultrasonic parameters. The Hugoniot data and finite strain extrapolations are similar at low pressures, where thermal effects are minor. At high pressure, differences in velocities and temperatures along the isentrope and Hugoniot are used to constrain temperature coefficients of velocity. Temperature derivatives at high pressure for $\mathrm{Al}, \mathrm{Cu}, \mathrm{Ta}$, and forsterite decrease in their absolute values by factors of 2 to 8. These results are consistent with expected values based on thermodynamic assumptions. Sound velocities in shockmelted metals and alkali halides have also been investigated. The Hugoniot velocities of the liquids are similar to or faster than calculated isentrope values based on low-pressure solid properties despite the fact that the Hugoniot states can be hotter by thousands of $\mathrm{K}$.

For forsterite, the average value of $\left|\left(\partial V_{P} / \partial T\right)_{P}\right|$ in the high-pressure region $(100-143 \mathrm{GPa})$ is $0.1 \pm 0.1 \mathrm{~m} / \mathrm{s} / \mathrm{K}$, a factor of 5 reduction relative to ambient-pressure laboratory values for forsterite and periclase. While uncertainties are larger for this material because of limited data and questions concerning strength and equilibrium, we note that the forsterite high-pressure phases behave in an analogous manner to the metals for which data and material characterization are more abundant.

These results have a number of geophysical implications. Using the high-pressure phases of forsterite as a lower mantle analogue, the temperature coefficient of compressional velocity is estimated to decrease in magnitude by a factor of about 5 from the Earth's surface to the base of the mantle. An upper bound estimate of $\left|\left(\partial V_{P} / \partial T\right)_{P}\right|$ based on thermoelastic properties for the Earth yields $\left|\left(\partial V_{P} / \partial T\right)_{P}\right|=$ $0.28 \mathrm{~m} / \mathrm{s} / \mathrm{K}$ at $1 \mathrm{Mbar}$, nearly a factor of 2 decrease relative to ambient-pressure values. A $50 \%$ reduction in the magnitude of the temperature coefficients of the elastic moduli across the mantle could reconcile these two estimates. Our results are also consistent with thermodynamic estimates of $\left(\partial V_{B} / \partial T\right)_{P}$ in the deep lower mantle.

The observed pressure dependence of $\left|\left(\partial V_{P} / \partial T\right)_{P}\right|$ is inconsistent with the results of residual spheres studies which largely sample the mantle above a depth of $1000 \mathrm{~km}$. Our results suggest velocity heterogeneities associated with subducting slabs should decrease with depth and this would make any lower mantle slab extensions more difficult to image by this method. The quantity $\left(\partial V_{P} / \partial \rho\right)_{P}$ is important in relating seismically observed velocity anomalies to density heterogeneities required for dynamic Earth models. Combining the forsterite data with mineral physics estimates of thermal expansivity, we obtain $\left(\partial V_{P} / \partial \rho\right)_{P}=$ $2(\mathrm{~km} / \mathrm{s}) /\left(\mathrm{g} / \mathrm{cm}^{3}\right)$ at $P>100 \mathrm{GPa}$, with possible values ranging from 0 to 8 . This overlaps the range of values obtained from most geoid models. Combining $\left|\left(\partial V_{P} / \partial T\right)_{P}\right|$ with velocity anomalies from seismic tomography, the magnitude of long-wavelength heterogeneities in the deep lower mantle, if thermal in origin, is $120 \pm 100 \mathrm{~K}$. In the $D^{\prime \prime}$ region, the anomalies must be a factor of 3-4 larger to satisfy the seismic data. The decrease in the observed magnitude of $P$ velocity variations between the upper mantle and the lower mantle can, except near boundaries, be explained by the decrease in the temperature coefficient of velocity and does not necessarily require thermal anomalies to be smaller in the lower mantle. The large value of $\left(\partial \ln V_{S} / \partial \ln V_{P}\right)_{P}$ $\geq 2$ observed in tomographic and free oscillation studies of the lower mantle cannot be explained by the shock data for homogeneous materials. Rather we require temperature variations of $120 \pm 100 \mathrm{~K}$ on length scales of $10^{4} \mathrm{~km}$ in the lower mantle and it is suggested elsewhere that the high value of $\left(\partial \ln V_{S} / \partial \ln V_{P}\right)_{P}$ could result from small fractions of partial melt [Duffy and Ahrens, 1992b].

Acknowledgments. We thank Don L. Anderson for many interesting discussions and suggestions. Critical reviews by Donald Isaak, Mark Richards, David Stevenson, J. Huw Davies, and an anonymous reviewer improved the manuscript considerably. The research was supported by NSF grant EAR-8816669. Division of Geological and Planetary Sciences, California Institute of Technology, contribution 4952 .

\section{REFERENCES}

Agnon, A., and M. S. T. Bukowinski, $\delta$, at high pressure and $d \ln V_{g} / d \ln V_{p}$ in the lower mantle, Geophys. Res. Lett., 17 , 1149-1152, 1990.

Ahrens, T. J., Shock wave techniques for geophysics and planetary physics, in Methods of Experimental Physics, vol. 24, edited by C. G. Sammis and T. L. Henyey, PP. 185-235, Academic, San Diego, Calif., 1987.

Al'tshuler, L. V., S. B. Kormer, M. I. Brazhnik, L. A. Vladimirov, M. P. Speranskaya, and A. I. Funtikov, The isentropic compressibility of aluminum, copper, lead, and iron at high pressures, Sov. Phys. JETP, Engl. Transl., 11, 761-775, 1960.

Anderson, D. L., A seismic equation of state, II, Shear properties and thermodynamics of the lower mantle, Phys. Earth Planet. Inter., 45, 307-323, 1987.

Anderson, D. L., Temperature and pressure derivatives of elastic constants with applications to the mantle, J. Geophys. Res., 93, 4688-4700, 1988.

Anderson, O. L., and T. Goto, Measurement of elastic constants of mantle-related minerals at temperatures up to 1800 K, Phys. Earth Planet. Inter., 55, 241-253, 1989.

Anderson, O. L., A. Chopelas, and R. Boehler, Thermal expansion versus pressure at constant temperature: $A$ reexamination, Geophys. Res. Lett., 17, 685-688, 1990.

Asay, J. R., and L. C. Chhabildas, Determination of the shear strength of shock compressed 6061-T6 aluminum, in Shock Waves and High Strain-Rate Phenomena in Metals, edited by M. A. Meyers, and L. E. Murr, pp. 417-431, Plenum, New York, 1981.

Asay, J. R., L. C. Chhabildas, and D. P. Dandekar, Shear strength of shock-loaded polycrystalline tungsten J. Appl. Phys., 51, 4774-4783, 1980 .

Asay, J. R., L. C. Chhabildas, G. I. Kerley, and T. G. Trucano, High pressure strength of shocked aluminum, in Shock Waves in Condensed Matter - 1985 , edited by Y. M. Gupta, pp. 145149, Plenum, New York, 1986.

Barker, L. M., and R. E. Hollenbach, Laser interferometer for measuring high velocities of any reflecting surface, J. Appl. Phys., 49, 4669-4675, 1972.

Bassett, W., T. Takahashi, H. Mao, and J. Weaver, Pressure induced phase transition in $\mathrm{NaCl}, J$. Appl. Phys., 99, 319-325, 1968.

Birch, F., The effect of pressure upon the elastic properties of isotropic solids according to Murnaghan's theory of finite strain, J. Appl. Phys., 9, 279-288, 1938.

Birch, F., Elasticity and constitution of the Earth's interior, $J$. Geophys. Res., 57, 227-286, 1952.

Birch, F., Thermal expansion at high pressures, J. Geophys. Res., $79,817-819,1968$.

Birch, F., Finite strain isotherm and velocities for single-crystal and polycrystalline $\mathrm{NaCl}$ at high pressures and $300 \mathrm{~K}, J$. Geophys. Res., 83, 1257-1268, 1978.

Boness, D. A., and J. M. Brown, Time-resolved optical spectroscopy of shock-compressed fluid alkali haides, in Shock Waves in Condensed Matter - 1989, edited by S. C. Schmidt, J. N. Johnson, and L. W. Davison, pp. 863-866, NorthHolland, New York, 1990. 
Boness, D. A., J. M. Brown, and J. W. Shaner, Rarefaction velocities in shocked lead, in Shock Waves in Condensed Matter 1987, edited by S. C. Schmidt and N. C. Holmes, pp. 115-118, North-Holland, New York, 1988.

Boyd, T. M., and K. C. Creager, The geornetry of Aleutian subduction: Three-dimensional seismic imaging, J. Geophys. Res., 96, 2267-2291, 1991 .

Brown, J. M., and R. G. McQueen, Phase transitions, Grüneisen parameter, and elasticity for shocked iron between $77 \mathrm{GPa}$ and $400 \mathrm{GPa}, J$. Geophys. Res., 91, 7485-7494, 1986.

Brown, J. M., and J. W. Shaner, Rarefaction velocities in shocked tantalum and the high pressure melting point, in Shock Waves in Condensed Matter - 1983, edited by J. R. Asay, R. A. Graham, and G. K. Straub, pp. 91-94, Elsevier, New York, 1984.

Brown, J. M., M. D. Furnish, and R. G. McQueen, Thermodynamics for $(\mathrm{Mg}, \mathrm{Fe})_{2} \mathrm{SiO}_{4}$ from the Hugoniot, in High Pressure Research in Mineral Physics, edited by M. Manghnani and Y. Syono, pp. 373-384, AGU, Washington, D. C., 1987.

Chhabildas, L. C., and J. R. Asay, Time-resolved wave profile measurements in copper to megabar pressures, in High Pressure in Research and Industry, 8th AIRAPT Conf., edited by C. M. Backman, T. Johannisson, and L. Tegman, Pp. 183-189, 1982.

Chhabildas, L. C., J. R. Asay, and L. M. Barker, Shear strength of tungsten under shock- and quasi-isentropic loading to 250 GPa, Rep. SAND88-0306, Sandia Natl. Lab., Albuquerque, N. M., 1988.

Chopelas, A., and R. Boehler, Thermal expansion measurements at very high pressure, systematics, and a case for a chemically homogeneous mantle, Geophys. Res. Lett., 16, 1347$1350,1989$.

Clayton, R. W., and R. P. Comer, A tomographic analysis of mantle heterogeneities from body wave travel times (abstract), Eos Trans $A G U, 64,776,1983$.

Creager, K. C., and T. H. Jordan, Slab penetration into the lower mantle beneath the Marianas and other island arcs of the northwest Pacific, J. Geophys. Res., 91, 3573-3580, 1986.

Davies, G. F., and A. M. Dziewonski, Homogeneity and constitution of the Earth's lower mantle and outer core, Phys. Earth Planet. Inter, 10, 336-343, 1975.

Davies, J. H., Some problems in mantle structure and dynamics, Ph.D. thesis, 315 pp., California Institute of Technology, Pasadena, 1990.

Duffy, T. S., and T. J. Ahrens, Hugoniot sound velocities and finite strain theory, in Shock Waves in Condensed Matter 1989 , edited by S. C. Schmidt, J. N. Johnson, and L. W. Davison, pp. 91-94, North-Holland, New York, 1990.

Duffy, T. S., and T. J. Ahrens, Hugoniot sound velocities in metals with applications to the Earth's inner core, in HighPressure Research in Mineral Physics: Applications to Earth and Planetary Sciences, Terra Scientific, Tokyo, in press, $1992 a$.

Duffy, T. S., and T. J. Ahrens, Lateral variations in lower mantle seismic velocity, in High-Pressure Research in Mineral Physics: Applications to Earth and Planetary Sciences, Terra Scientific, Tokyo, in press, $1992 b$.

Dziewonski, A. M., Mapping the lower mantle: Determination of lateral heterogeneity in $\mathrm{P}$ velocity up to degree and order 6 , J. Geophys, Res., 89, 5929-5952, 1984.

Dziewonski, A. M., and D. L. Anderson, Preliminary reference Earth model, Phys. Earth Planet. Inter., 25, 297-356, 1981.

Dziewonski, A. M., and J. H. Woodhouse, Global images of the Earth's interior, Science, 236, 37-48, 1987.

Fischer, K. M., T. H. Jordan, and K. C. Creager, Seismic constraints on the morphology of deep slabs, J. Geophys. Res., 99, 4773-4783, 1988.

Fischer, K. M., K. C. Creager, and T. H. Jordan, Mapping the Tonga slab, J. Geophys. Res., 96, 14,403-14,427, 1991.

Forte, A. M., and W. R. Peltier, Plate-tectonics and aspherical Earth structure - The importance of poloidal-toroidal coupling, J. Geophys. Res., 92, 3645-3679, 1987.

Forte, A. M., and W. R. Peltier, Mantle convection and core mantle boundary topography - Explanations and implications, Tectonophysics, 187, 91-116, 1991.

Furnish, M. D., Measuring the dynamic compression and release behavior of the paintbrush and tunnel bed (NTS) tuffs over the range 1-13 GPa, Rep. SAND90-1917, Sandia Natl. Lab., Albuquerque, N. M., 1990.

Grady, D. E., Processes occurring in shock wave compression of rocks and minerals, in High Pressure Research in Mineral Physics, edited by M. Manghnani and Y. Syono, pp. 389-438, Academic, New York, 1977.

Gudmundsson, O., J. H. Davies, and R. W. Clayton, Stochastic analysis of global travel time data: Mantle heterogeneity and random errors in the ISC data, Geophys. J. Int., 102, 25-43, 1990.

Hager, B. H., and R. W. Clayton, Constraints on the structure of mantle convection using seismic observations, flow models, and the geoid, in Mantle Convection: Plate Tectonics and Global Dynamics, edited by W. R. Peltier, pp. 657-764, Gordon and Breach, New York, 1989.

Hager, B. H., and M. A. Richards, Long-wavelength variations in Earth's geoid: Physical models and dynamical implications, Philos. Trans. R. Soc. London, Ser. A, 328, 309-327, 1989.

Hager, B. H., R. W. Clayton, M. A. Richards, R. P. Comer, and A. M. Dziewonski, Lower mantle heterogeneity, dynamic topography, and the geoid, Nature, 913, 541-545, 1985.

Hixson, R. S., D. A. Boness, J. W. Shaner, and J. A. Moriarty, Acoustic velocities and phase transitions in molybdenum under shock compression, Phys. Rev. Lett., 62, 637-640, 1989.

Ho, P. S., and A. L. Ruoff, Pressure dependence of the elastic constants for aluminum from 77 to $300 \mathrm{~K}, J$. Appl. Phys., 40 , 3151-3156, 1969.

Hu, J., F. Jing, and J. Cheng, Sound velocities at high pressures and shock-melting of copper, Chinese J. High Pressure Phys., 3, 187-197, 1989.

Isaak, D. G., O. L. Anderson, and T. Goto, Measured elastic moduli of single crystal $\mathrm{MgO}$ up to $1800 \mathrm{~K}$, Phys. Chem. Miner., $16,704-713,1989 a$.

Isaak, D. G., O. L. Anderson, and T. Goto, Elasticity of singlecrystal forsterite measured to $1700 \mathrm{~K}, J$. Geophys. Res., 94, 5895-5906, $1989 b$.

Isaak, D. G., R. E. Cohen, and M. J. Mehl, Calculated elastic and thermal properties of $\mathrm{M}_{\mathrm{g}} \mathrm{O}$ at high pressures and temperatures, J. Geophys. Res., 95, 7055-7067, 1990.

Jeanloz, R., and E. Knittle, Reduction of mantle and core properties to a standard state by adiabatic compression, in Chemistry and Physics of the Terrestrial Planets, edited by S. K. Saxena, pp. 275-305, Springer-Verlag, Berlin, 1986.

Jordan, T. H., A. L. Lerner-Lam, and K. C. Creager, Seismic imaging of boundary layers and deep mantle convection, in Mantle Convection: Plate Tectonics and Global Dynamics, edited by W. R. Peltier, pp. 97-202, Gordon and Breach, New York, 1989.

Katahara, K. W., M. H. Manghnani, and E. S. Fisher, Pressure derivatives of the elastic moduli of BCC Ti-V-Cr, Nb-Mo, and Ta-W alloys, J. Phys. F: Metal Phys., 9, 773-790, 1979.

Knittle, E., and R. Jeanloz, Synthesis and equation of state of $(\mathrm{Mg}, \mathrm{Fe}) \mathrm{SiO}_{3}$ perovskite to over 100 Gigapascals, Science, 235, 668-670, 1987.

Knittle, E., R. Jeanloz, and G. L. Smith, Thermal expansion of silicate perovskite and stratification of the Earth's mantle, Nature, 319, 214-216, 1986.

Li, X. D., D. Giardini, and J. H. Woodhouse, The relative amplitudes of mantle heterogeneity in P-velocity, S-velocity, and density from free-oscillation data, Geophys. J., 105, 649-658, 1991.

Lipkin, J., and J. R. Asay, Reshock and release of shockcompressed 6061-T6 aluminum, J. Appl. Phys., 48, 182-189, 1977 .

Lyzenga, G. A., and T. J. Ahrens, Shock temperature measurements in $\mathrm{Mg}_{2} \mathrm{SiO}_{4}$ and $\mathrm{SiO}_{2}$ at high pressure, Geophys. Res. Lett., 7, 141-144, 1980.

Machetel, P., Short wavelength lower mantle seismic velocity variations, Geophys. Res. Lett., 17,, 1145-1148, 1990.

McNutt, M. K., and A. V. Judge, The superswell and mantle dynamics beneath the south Pacific, Science, 248, 969-975, 1990.

McQueen, R. G., S. P. Marsh, J. W. Taylor, J. N. Fritz, and W. J. Carter, The equation of state of solids from shock wave studies, in High-Velocity Impact Phenomena, edited by R. Kinslow, pp. 294-419, Academic, San Diego, Calif., 1970.

McQueen, R. G., J. W. Hopson, and J. N. Fritz, Optical tech- 
nique for determining rarefaction wave velocities at very high pressures, Rev. Sci. Instrum., 59, 245-250, 1982.

McQueen, R. G., J. N. Fritz, and C. E. Morris, The velocity of sound behind strong shock waves in $2024 \mathrm{Al}$, in Shock Waves in Condensed Matter - 1983, edited by J. R. Asay, R. A. Graham, and G. K. Straub, pp. 95-98, Elsevier, New York, 1984.

Neal, T., Dynamic determination of the Grüneisen coefficient in aluminum and aluminum alloys for densities up to $6 \mathrm{Mg} / \mathrm{m}^{3}$, Phys. Rev. B, 14, 5172-5181, 1976.

Reynard, B., and G. D. Price, Thermal expansion of minerals at high pressures - A theoretical study, Geophys. Res. Lett., 17, 689-692, 1990.

Ricard, Y., C. Vigny, and C. Froidevaux, Mantle heterogeneities, geoid, and plate motion - a Monte-Carlo inversion, J. Geophys. Res., 94, 13,739-13,754, 1989.

Richards, M. A., and B. H. Hager, Effects of lateral viscosity variations on long-wavelength geoid anomalies and topography, $J$. Geophys. Res., 94, 10,299-10,313, 1989.

Sammis, C., D. Anderson, and T. Jordan, Application of isotropic finite strain theory to ultrasonic and seismological data, $J$. Geophys. Res., 75, 4478-4480, 1970.

Schmunk, R. E., and C. S. Smith, Pressure derivatives of the elastic constants of aluminum and magnesium, J. Phys. Chem. Solids, 9, 100-112, 1959.

Schwartz, S. Y., T. Lay, and S. P. Grand, Seismic imaging of subducted slabs: Trade-offs with deep path and near-receiver effects, Geophys. Res. Lett., 18, 1265-1268, 1991.

Shaner, J. W., J. M. Brown, and R. G. McQueen, Melting of metals above $100 \mathrm{GPa}$, in High Pressure in Science and Technology, 22, edited by C. Homan, R. K. MacCrone, and E. Whalley, pp. 137-141, North-Holland, New York, 1984.

Shaner, J. W., R. S. Hixson, M. A. Winkler, D. A. Boness, and J. M. Brown, Birch's law for fluid metals, in Shoc? Waves in Condensed Matter - 1987, edited by S. C. Schmidi and N. C. Holmes, pp. 135-138, North-Holland, New York, 1 188.

Simmons, G., and H. Wang, Single Crystal Elastic Constants and Calculated Aggregate Properties: A Handbook, 370 pp. MIT Press, Cambridge, Mass., 1971.

Sleep, N. H., Teleseismic P-wave transmission through slabs, Bull. Seismol. Soc. Am., 63, 1349-1373, 1973.
Spetzler, H. A. W., Effect of temperature and pressure on elastic properties of polycrystalline and single crystal $\mathrm{MgO}$, Ph.D. thesis, 246 pp., California Institute of Technology, Pasadena, 1969.

Stacey, F. D., A thermal model of the Earth, Phys. Earth Planet. Inter., 15, 341-348, 1977.

Stacey, F. D., and D. E. Loper, The thermal boundary-layer interpretation of D" and its role as a plume source, Phys. Earth Planet. Inter., 39, 45-55, 1983.

$\mathrm{Su}, \mathrm{W}$., and A. M. Dziewonski, Predominance of long-wavelength heterogeneity in the mantle, Nature, 352, 121-126, 1991.

Sumino, Y., and O. L. Anderson, Elastic constants of minerals, in Handbook of Physical Properties of Rocks, vol. III, edited by R. S. Carmichael, PP. 39-137, CRC Press, Boca Raton, Fla., 1984.

Swenson, C. A., J. W. Shaner, and J. M. Brown, Hugoniot overtake sound-velocity measurements of CsI, Phys. Rev. B, 39, 7924-7935, 1986.

Thomas, J. F., Third-order elastic constants of aluminum, Phys. Rev., 175, 955-962, 1968.

Wang, G., An empirical expression of elastic sound speed of materials at high pressure, Chinese J. High Pressure Phys., 2, 92-95, 1988.

Yeganeh-Haeri, A., D. J. Weidner, and E. Ito, Elasticity of $\mathrm{MgSiO}_{3}$ in the perovskite structure, Science, 249, 787-789, 1989.

Zharkov, V., and V. Kalinin, Equations of State for Solids at High Pressures and Temperatures, pp. 257, Consultants Bureau, New York, 1971.

Zhou, H. W., D. L. Anderson, and R. W. Clayton, Modeling of residual spheres for subduction zone earthquakes, 1, Apparent slab penetration signatures in the NW Pacific, J. Geophys. Res., 95, 6799-6827, 1990.

T. J. Ahrens and T. S. Duffy, Seismological Laboratory 252-21, California Institute of Technology, Pasadena, CA 91125.

(Received November 26, 1990; revised October 4, 1991; accepted October 17, 1991.) 